Annales Academiæ Scientiarum Fennicæ

Mathematica

Volumen 35, 2010, 439-472

\title{
LINEARIZATION MODELS FOR PARABOLIC DYNAMICAL SYSTEMS VIA ABEL'S FUNCTIONAL EQUATION
}

\author{
Mark Elin, Dmitry Khavinson, Simeon Reich and David Shoikhet \\ ORT Braude College, Department of Mathematics \\ P.O. Box 78, 21982 Karmiel, Israel; mark_elin@braude.ac.il \\ University of South Florida, Department of Mathematics \\ Tampa, FL 33620-5700, U.S.A.; dkhavins@cas.usf.edu \\ Technion - Israel Institute of Technology, Department of Mathematics \\ 32000 Haifa, Israel; sreich@tx.technion.ac.il \\ ORT Braude College, Department of Mathematics \\ P.O. Box 78, 21982 Karmiel, Israel; davs@braude.ac.il
}

\begin{abstract}
We study linearization models for continuous one-parameter semigroups of parabolic type. In particular, we introduce new limit schemes to obtain solutions of Abel's functional equation and to study asymptotic behavior of such semigroups. The crucial point is that these solutions are univalent functions convex in one direction. In a parallel direction, we find analytic conditions which determine certain geometric properties of those functions, such as the location of their images in either a half-plane or a strip, and their containing either a half-plane or a strip. In the context of semigroup theory these geometric questions may be interpreted as follows: is a given one-parameter continuous semigroup either an outer or an inner conjugate of a group of automorphisms? In other words, the problem is finding a fractional linear model of the semigroup which is defined by a group of automorphisms of the open unit disk. Our results enable us to establish some new important analytic and geometric characteristics of the asymptotic behavior of one-parameter continuous semigroups of holomorphic mappings, as well as to study the problem of existence of a backward flow invariant domain and its geometry.
\end{abstract}

\section{Introduction}

Let $\Delta$ be the open unit disk in the complex plane $\mathbf{C}$ and let $\Omega$ be a subset of C. By $\operatorname{Hol}(\Delta, \Omega)$ we denote the set of all holomorphic functions (mappings) from $\Delta$ into $\Omega$. Linearization models for continuous semigroups of holomorphic mappings in various settings have an extensive history, beginning with the study of continuous stochastic Markov branching processes (see, for example, [26]), Kolmogorov's backward equation in probability theory and functional differential equations. The linearization models for one-parameter semigroups have also proved to be very useful in the study of composition operators and their spectra (see, for example, [4], [15], [42], [16]). In the years since these classic works appeared, much more information has been found.

A deep investigation of the behavior of one-parameter semigroups near their boundary Denjoy-Wolff point based on geometric properties of the model obtained by Abel's functional equation has recently been conducted in [12, 13]. See also [11]. It

doi:10.5186/aasfm.2010.3528

2000 Mathematics Subject Classification: Primary 30C45, 30D05, 37F99, 47H20.

Key words: Abel's functional equation, asymptotic behavior, convex in one direction, generator, linearization model, semigroup of parapolic type. 
turns out that in those settings solutions of Abel's functional equation are univalent functions convex in one direction.

In a parallel direction, the class of functions convex in one direction has been studied by many mathematicians (see, for example, [27], [9], [8], [25] and [29]) as a subclass of the class of functions introduced by Robertson in [36]. Following this point of view, we study in the present paper several additional geometric and analytic properties of functions convex in one direction by using the asymptotic behavior of one-parameter semigroups near their attractive (or repelling) boundary fixed points.

In an opposite direction this study yields new information concerning some special (but very wide and important) classes of complex dynamical systems generated by holomorphic functions having fractional derivatives at their Denjoy-Wolff points.

Definition 1. A univalent function $h \in \operatorname{Hol}(\Delta, \mathbf{C})$ is said to be convex in the positive direction of the real axis if for each $z \in \Delta$ and $t \geq 0$, the point $h(z)+t$ belongs to $h(\Delta)$.

It is well known (see, for example, [37], p. 162) that for each $z \in \Delta$, the limit

$$
\lim _{t \rightarrow \infty} h^{-1}(h(z)+t)=: \zeta
$$

exists and belongs to $\partial \Delta$. Moreover, since the family $S=\left\{F_{t}\right\}_{t \geq 0}$ defined by

$$
F_{t}(z)=h^{-1}(h(z)+t)
$$

forms a one-parameter continuous semigroup of holomorphic self-mappings of $\Delta$, it follows from the continuous version of the Denjoy-Wolff Theorem (see [4], [33] and [38]) that the limit point $\zeta$ in (1) is unique and does not depend on $z \in \Delta$.

Without loss of generality we may set $\zeta=1$. We denote by $\Sigma[1]$ the class of functions convex in the positive direction of the real axis, normalized by the conditions

$$
\lim _{t \rightarrow \infty} h^{-1}(h(z)+t)=1 \text { and } h(0)=0 .
$$

In the reverse direction one can assign to each semigroup $S=\left\{F_{t}\right\}_{t \geq 0}$ of holomorphic self-mappings of $\Delta$ with a boundary Denjoy-Wolff point $\zeta=1$, a univalent function $h \in \operatorname{Hol}(\Delta, \mathbf{C})$ which is a common solution to Abel's functional equations

$$
h\left(F_{t}(z)\right)=h(z)+t, z \in \Delta, t \geq 0,
$$

and hence is convex in the positive direction of the real axis (see the proof of Proposition 3 below). The set $h(\Delta)$ is called a planar domain for $S$ and the pair $(h, h(\Delta))$ is said to be a linearization model for $S$.

To be more precise, we recall that by the Berkson-Porta theorem [4] (see also [1], [31], [32] and [35]), for each one-parameter continuous semigroup $S=\left\{F_{t}\right\}_{t \geq 0}$ of holomorphic self-mappings of $\Delta$, the limit

$$
f(z):=\lim _{t \rightarrow 0^{+}} \frac{z-F_{t}(z)}{t}
$$

exists. This limit function is called the infinitesimal generator of $S$. Moreover, the semigroup $S=\left\{F_{t}\right\}_{t \geq 0}$ can be reproduced as the solution of the Cauchy problem

$$
\left\{\begin{array}{l}
\frac{\partial u(t, z)}{\partial t}+f(u(t, z))=0 \\
u(0, z)=z
\end{array}\right.
$$


where we set $F_{t}(z):=u(t, z), t \geq 0, z \in \Delta$.

By $\mathscr{G}[1]$ we denote the class of functions which consists of all the holomorphic generators $f$ of continuous semigroups having the Denjoy-Wolff point $\zeta=1$. In this case $f$ admits the Berkson-Porta representation

$$
f(z)=-(1-z)^{2} p(z), \quad z \in \Delta,
$$

with $\operatorname{Re} p(z) \geq 0$ everywhere (see [4] and [38]).

In addition, if $S=\left\{F_{t}\right\}_{t \geq 0}$ is the semigroup generated by $f$ ( $S$ is defined via the Cauchy problem (6), where we set $\left.F_{t}(z):=u(t, z), t \geq 0, z \in \Delta\right)$, then $\lim _{t \rightarrow \infty} F_{t}(z)=1$ and for each $t \geq 0$,

$$
\angle \lim _{z \rightarrow 1} \frac{\partial F_{t}(z)}{\partial z}=e^{-t \beta}
$$

where $\beta:=\angle \lim _{z \rightarrow 1} \frac{f(z)}{z-1}=\angle \lim _{z \rightarrow 1} f^{\prime}(z) \geq 0$ (see, for example, [20] and [12]).

If $\beta>0$, then $S$ and its generator $f$ are said to be of hyperbolic type. Otherwise, that is, if $\beta=0$, the semigroup $S$ and its generator $f$ are said to be of parabolic type. Conversely, it is shown in [20] (see also [39] and [12]) that if $f$ is a holomorphic generator on $\Delta$ such that the angular limit

$$
\angle \lim _{z \rightarrow 1} \frac{f(z)}{z-1}=: f^{\prime}(1)
$$

exists finitely with $\operatorname{Re} f^{\prime}(1) \geq 0$, then $f^{\prime}(1)$ is, in fact, a real number, $f$ has no null points in $\Delta$ and belongs to $\mathscr{G}[1]$. This, in turn, implies (see Section 1 for details) that the formula

$$
h(z)=-\int_{0}^{z} \frac{d z}{f(z)}
$$

establishes a one-to-one correspondence between the classes $\mathscr{G}[1]$ and $\Sigma[1]$.

In this paper we are interested in finding simple analytic conditions which determine certain geometric properties of functions $h$ in the class $\Sigma[1]$, such as the location of the image $h(\Delta)$ in either a half-plane or a strip, and its containing either a half plane or a strip.

In the context of semigroup theory these geometric questions may be interpreted as follows: Is a given one-parameter continuous semigroup either an outer or an inner conjugate of a group of automorphisms (see the definition below)? In other words, the problem is finding a fractional linear model of the semigroup which is defined by a group of automorphisms of $\Delta$. To be more precise, we need the following definition.

Definition 2. Let $S=\left\{F_{t}\right\}_{t \geq 0}$ be a one-parameter continuous semigroup of holomorphic self-mappings of $\Delta$.

- A univalent mapping $\psi: \Delta \mapsto \Delta$ is called an outer conjugator of $S$ if there is a one-parameter semigroup $\left\{G_{t}\right\}_{t \geq 0} \subset \operatorname{Hol}(\Delta, \Delta)$ of linear fractional transformations of $\Delta$ such that

$$
\psi \circ F_{t}=G_{t} \circ \psi \text {. }
$$

- A univalent mapping $\varphi: \Delta \mapsto \Delta$ is called an inner conjugator of $S$ if there is a one-parameter continuous semigroup $\left\{G_{t}\right\}_{t \geq 0} \subset \operatorname{Hol}(\Delta, \Delta)$ of linear fractional 
transformations of $\Delta$ such that

$$
F_{t} \circ \varphi=\varphi \circ G_{t} .
$$

Note that by the Linear Fractional Model theorem (see [5] and [37]) for each holomorphic self-mapping there exists a conjugating function $G: \Delta \mapsto \mathbf{C}$ which is not necessarily a self-mapping of $\Delta$. However, in our considerations the main point in Definition 2 is that the conjugators $\varphi$ and $\psi$ are self-mappings of $\Delta$.

It is rather a transparent fact (see Sections 2 and 3 for details) that if $S=\left\{F_{t}\right\}_{t \geq 0}$ is a semigroup with a boundary Denjoy-Wolff point $\zeta \in \partial \Delta($ say $\zeta=1)$ and $h$ is the common solution to Abel's equations (4) normalized by (3), then an outer (respectively, inner) conjugator of $S$ exists if and only if $h(\Delta)$ lies in (respectively, contains) a half-plane $\Pi$. In both cases the conjugate semigroup $\left\{G_{t}\right\}_{t \geq 0}$ of linear fractional transformations (see formulas (8) and (9)) is a group of automorphisms of $\Delta$ if and only if $\Pi$ is horizontal, i.e., its boundary is parallel to the real axis.

The latter situation is of special interest for inner conjugation since equation (9) means that the semigroup $S=\left\{F_{t}\right\}_{t \geq 0}$ forms a group of automorphisms of the domain $\varphi(\Delta)$ which is called a backward flow invariant domain for $S$ (see [24]). We will concentrate on this issue in Section 3 for semigroup generators which are fractionally differentiable at their boundary Denjoy-Wolff points. In the meantime we need the following observation.

It can be shown (see [22] and Lemma 6 below) that if $h$ belongs to $\Sigma[1]$, then the angular limit

$$
\mu:=\angle \lim _{z \rightarrow 1}(1-z) h^{\prime}(z)
$$

exists and belongs to $(0, \infty]$. It turns out that this limit $\mu$ is finite if and only if $|\operatorname{Im} h(z)|$ is bounded. Moreover, the number $\pi \mu$ is the width of the minimal horizontal strip which contains $h(\Delta)$.

If $\mu$ in (10) is infinite, it may happen that for some $\alpha>0$, the angular limit

$$
\angle \lim _{z \rightarrow 1}(1-z)^{1+\alpha} h^{\prime}(z)=: \mu
$$

or even the unrestricted limit

$$
\lim _{\substack{z \rightarrow 1 \\ z \in \Delta}}(1-z)^{1+\alpha} h^{\prime}(z)=: \mu
$$

exist finitely and are different from zero. The latter condition, for example, follows from the geometric property of $h(\Delta)$ to have a Dini smooth corner of opening $\pi \alpha$ at infinity for some $\alpha \in(0,2]$ (see [30], p. 54). Therefore it is natural to consider the subclasses $\Sigma_{A}^{\alpha}[1]$, respectively, $\Sigma^{\alpha}[1]\left(\Sigma^{\alpha}[1] \subset \Sigma_{A}^{\alpha}[1]\right), \alpha \geq 0$, which consist of those $h \in \Sigma[1]$ satisfying (11), respectively, (12).

We will first show that if $\Sigma_{A}^{\alpha}[1] \neq \varnothing$, then $\alpha$ must belong to the interval $[0,2]$. Furthermore, if $\alpha>0$ and $h \in \Sigma^{\alpha}[1]$, then the boundedness from above (or from below) of $\operatorname{Im} h(z)$ implies some necessary simple relations between the numbers $\alpha$ and $\mu$ in (12). Note that if $\alpha>0$, then $\operatorname{Im} h(z)$ cannot be bounded from both above and below. In fact, $\Sigma[1] \backslash \bigcup_{\alpha \geq 0} \Sigma_{A}^{\alpha}[1] \neq \varnothing$, as the example of $h(z)=\int_{0}^{z} \frac{d z}{(1-z)^{2} \log \frac{2}{1-z}}$ shows. At the same time, for each $0 \leq \alpha \leq 2$, the class $\Sigma_{A}^{\alpha}[1]$ is dense in $\Sigma[1]$ and contains numerous important examples. 
Finally, we would like to emphasize that these relations, as well as the geometric properties of these classes, are connected to the asymptotic behavior of the semigroups defined by (2). In particular, for these classes we solve in the affirmative the common slope conjecture suggested in [11] (see Theorems 21 and 23 and Corollary 37). Also, some special situations arise when $\alpha$ attains its integer values $\{0,1,2\}$. In particular, in Theorem 23 , a criterion of the so-called strongly tangential convergence of the semigroup trajectories is given.

\section{Subordination theorems (outer conjugation)}

2.1. Auxiliary results. Analytic descriptions of functions convex in one direction have been presented by many mathematicians using different methods of classical geometric function theory including the prime ends theory and Julia's lemma (see, for example, [36], [27], [9], [8], [25] and [29]). For completeness we begin with the following description of the class $\Sigma[1]$, which is a slight generalization of those given in [27], [9], [8], and [29], and is based on the relations between this class and the class $\mathscr{G}[1]$.

Proposition 3. A function $h \in \operatorname{Hol}(\Delta, \mathbf{C}), h \neq 0$, with $h(0)=0$, belongs to $\Sigma[1]$ if and only if

$$
\operatorname{Re}\left[(1-z)^{2} h^{\prime}(z)\right] \geq 0
$$

and if and only if the function $f \in \operatorname{Hol}(\Delta, \Omega)$, defined by $f(z)=-\frac{1}{h^{\prime}(z)}$, belongs to $\mathscr{G}[1]$.

Proof. Indeed, if $h \in \Sigma[1]$, then $S=\left\{F_{t}\right\}_{t \geq 0}$, where $F_{t}$ is defined by (2), is a one-parameter continuous semigroup with the Denjoy-Wolff point $\zeta=1$. Therefore, by the Berkson-Porta theorem [4], its infinitesimal generator

$$
f(z):=\lim _{t \rightarrow 0^{+}} \frac{z-F_{t}(z)}{t}
$$

admits the representation

$$
f(z)=-(1-z)^{2} p(z)
$$

with

$$
\operatorname{Re} p(z) \geq 0 \text {. }
$$

On the other hand, differentiating $(2)$ at $t=0^{+}$, we obtain

$$
f(z)=-\frac{1}{h^{\prime}(z)} \text {. }
$$

Thus (15) and (16) imply (13).

Conversely, assume now that $h \in \operatorname{Hol}(\Delta, \mathbf{C}), h \neq 0$, with $h(0)=0$, satisfies (13). If $\operatorname{Re}\left[(1-z)^{2} h^{\prime}(z)\right]=0$, then $h(z)=\frac{i b z}{1-z}$ for some $b \in \mathbf{R}$. Moreover, $b \neq 0$ since by the hypothesis $h$ is not identically zero; so $h$ clearly belongs to $\Sigma[1]$. In this case the function $f \in \operatorname{Hol}(\Delta, \Omega)$, defined by $f(z)=-\frac{1}{h^{\prime}(z)}$, is a generator of a group of parabolic automorphisms of $\Delta$, hence belongs to $\mathscr{G}[1]$. 
So we may assume that

$$
\operatorname{Re}\left[(1-z)^{2} h^{\prime}(z)\right]>0 .
$$

In this case, using the convex function $g(z)=\frac{z}{1-z}$, we have

$$
\operatorname{Re}\left[\frac{h^{\prime}(z)}{g^{\prime}(z)}\right]>0
$$

which means that $h$ is close-to-convex. Hence it must be univalent (see [18], Theorem 2.17 , p. 47 ).

In addition, we know that the function $f \in \operatorname{Hol}(\Delta, \mathbf{C})$ defined by

$$
f(z)=-\frac{1}{h^{\prime}(z)}
$$

admits the Berkson-Porta representation (15) with (16). Therefore, the Cauchy problem

$$
\left\{\begin{array}{l}
\frac{\partial u(t, z)}{\partial t}+f(u(t, z))=0, \\
u(0, z)=z
\end{array}\right.
$$

is solvable with $|u(t, z)|<1$ for each $z \in \Delta$ and all $t \geq 0$. Moreover, it follows from (17) and (18) that

$$
h^{\prime}(u(t, z)) \cdot \frac{\partial u(t, z)}{\partial t}=1
$$

for all $z \in \Delta$ and $t \geq 0$.

Integrating this equality, we get

$$
h(u(t, z))=h(z)+t \in h(\Delta)
$$

for all $z \in \Delta$ and $t \geq 0$. Hence $h$ is indeed convex in the positive direction of the real axis.

Finally, again by the Berkson-Porta theorem, we have $\lim _{t \rightarrow \infty} u(t, z)=1$. Hence $h \in \Sigma[1]$ and the proof is complete.

Thus, we have shown that the formula

$$
h(z)=-\int_{0}^{z} \frac{d z}{f(z)}
$$

determines a one-to-one correspondence between the classes $\mathscr{G}[1]$ and $\Sigma[1]$.

For $h \in \Sigma[1]$ we assume now that $h(\Delta)$ lies in a horizontal half-plane $\Pi=h_{1}(\Delta)$, where $h_{1}: \Delta \mapsto \mathbf{C}$ is defined by

$$
h_{1}(z)=\frac{i b z}{1-z}
$$

for some nonzero $b \in \mathbf{R}$. Consider the mapping $\psi: \Delta \mapsto \Delta$ defined by

$$
\psi(z)=h_{1}^{-1}(h(z)), \quad \psi(0)=0,
$$

or explicitly

$$
\psi(z)=\frac{h(z)}{i b+h(z)} .
$$


Define a group of (parabolic) automorphisms of $\Delta$ by

$$
\begin{aligned}
G_{t}(z) & =h_{1}^{-1}\left(h_{1}(z)+t\right)=\frac{h_{1}(z)+t}{i b+h_{1}(z)+t}, \quad t \in \mathbf{R}, \\
& =\frac{\frac{i b z}{1-z}+t}{i b+\frac{i b z}{1-z}+t}=\frac{i b z+t(1-z)}{i b+t(1-z)} .
\end{aligned}
$$

It is clear that

$$
\lim _{t \rightarrow \pm \infty} G_{t}(z)=1
$$

Now for $t \geq 0$ we have by $(21)$

$$
G_{t}(\psi(z))=h_{1}^{-1}\left(h_{1}(\psi(z))+t\right)=h_{1}^{-1}(h(z)+t)=h_{1}^{-1}\left(h\left(F_{t}(z)\right)\right)=\psi\left(F_{t}(z)\right),
$$

where $F_{t}(z)=h^{-1}(h(z)+t)$. Thus, for $S=\left\{F_{t}\right\}_{t \geq 0}$ generated by $f$, defined by (17), there is a group $\left\{G_{t}\right\}_{t \in \mathbf{R}}$ of parabolic automorphisms of $\Delta$, such that $S$ is an outer conjugate of $\left\{G_{t}\right\}_{t>0}$ by $\psi: \Delta \mapsto \Delta$. Moreover, the domain $\Omega=\psi(\Delta)$ is invariant for the semigroup $\left\{G_{t}\right\}_{t \geq 0}$.

Conversely, let $\left\{G_{t}\right\}_{t \geq 0}$ be a group of parabolic automorphisms of $\Delta$ such that $\lim _{t \rightarrow \pm \infty} G_{t}(z)=1$, and assume that there is a holomorphic Riemann mapping $\psi$ of $\Delta$ into $\Delta$ (we may assume that $\psi(0)=0$ ) such that

$$
G_{t}(\psi(z))=\psi\left(F_{t}(z)\right)
$$

for all $z \in \Delta$ and $t \geq 0$.

Differentiating (22) with respect to $t \geq 0$, we have

$$
g(\psi(z))=\psi^{\prime}(z) \cdot f(z),
$$

where $g$ and $f$ are generators of $\left\{G_{t}\right\}_{t \geq 0}$ and $\left\{F_{t}\right\}_{t \geq 0}$, respectively. Explicitly, $g(z)$ can be calculated as follows:

$$
\begin{aligned}
g(z) & =\lim _{t \rightarrow 0^{+}} \frac{z-G_{t}(z)}{t}=\lim _{t \rightarrow 0} \frac{z-\frac{i b z+t(1-z)}{i b+t(1-z)}}{t} \\
& =\lim _{t \rightarrow 0} \frac{i b z+t z(1-z)-i b z-t(1-z)}{t(i b+t(1-z))} \\
& =\lim _{t \rightarrow 0^{+}} \frac{t(1-z)(z-1)}{t(i b+t(1-z))}=-\frac{(1-z)^{2}}{i b} .
\end{aligned}
$$

Since $f(z)=-\frac{1}{h^{\prime}(z)}$, we get from (23) and (24) that

$$
h^{\prime}(z)=\frac{\psi^{\prime}(z) i b}{(1-\psi(z))^{2}} .
$$

Integrating (25) from 0 to $z$, we obtain

$$
h(z)=\frac{i b \psi(z)}{1-\psi(z)} .
$$


Now, setting $w=\psi(z) \in \Omega$, we get from (26) that

$$
h\left(\psi^{-1}(w)\right)=\frac{i b w}{1-w}=: h_{1}(w) .
$$

Since this function can be extended to the whole disk $\Delta$ and $h_{1}(\Delta)$ is a horizontal halfplane, equality (26) means that $h(\Delta) \subset h_{1}(\Delta)$. Thus we have proved the following assertion.

Proposition 4. Let $\left\{F_{t}\right\}_{t>0}$ be a semigroup with the Denjoy-Wolff point $z=1$, such that $F_{t}(z)=h^{-1}(h(z+t)), h \in \Sigma[1]$. Then $h(\Delta)$ lies in a horizontal half-plane if and only if there is a group $\left\{G_{t}\right\}_{t \in \mathbf{R}}$ of parabolic automorphisms of $\Delta$ having 1 as the Denjoy-Wolff point and a conformal self-mapping $\psi$ of $\Delta$ such that for all $t \geq 0$, the semigroup $\left\{F_{t}\right\}$ outer conjugates with $\left\{G_{t}\right\}$ :

$$
\psi\left(F_{t}(z)\right)=G_{t}(\psi(z)), \quad t \geq 0 .
$$

Remark 5. In particular, each hyperbolic group can conjugate with a parabolic one. This can easily be shown if we translate our considerations to the right halfplane. Namely, let $\left\{\widehat{F}_{t}\right\}$ be the group of hyperbolic automorphisms of $\Pi_{+}=\{w \in$ C: $\operatorname{Re} w>0\}$ of the form

$$
\widehat{F}_{t}(w)=e^{t} w .
$$

Consider the univalent function $\widehat{\psi}: \Pi_{+} \mapsto \mathbf{C}$ given by

$$
\widehat{\psi}(w)=i \log w+r
$$

where $r>\pi / 2$. Then, for $w \in \Pi_{+}$we have $\frac{\pi}{2}+r>\operatorname{Re} \widehat{\psi}(w)=r-\arg w>0$. So, $\widehat{\psi}$ maps $\Pi_{+}$into a vertical strip $\widehat{\Omega} \subset \Pi_{+}$. In addition,

$$
\widehat{\psi}\left(\widehat{F}_{t}(z)\right)=i \log \left(e^{t} w\right)+r=i t+i \log w+r=i t+\widehat{\psi}(w) .
$$

Thus, if we define a group of parabolic automorphisms of $\Pi_{+}$by

$$
\widehat{G}_{t}(w)=w+i t
$$

we get that

$$
\widehat{\psi}\left(\widehat{F}_{t}(w)\right)=\widehat{G}_{t}(\widehat{\psi}(w)) .
$$

Applying now the Cayley transform $C: \Delta \mapsto \Pi^{+}, C(z)=\frac{1+z}{1-z}$, and setting $F_{t}=C^{-1} \circ \widehat{F}_{t} \circ C, G_{t}=C^{-1} \circ \widehat{G}_{t} \circ C$ and $\psi=C^{-1} \circ \widehat{\psi} \circ C$, one obtains the needed relation in the unit disk

$$
\psi\left(F_{t}(z)\right)=G_{t}(\psi(z)), \quad z \in \Delta .
$$

In this case $\Omega=C^{-1}(\widehat{\Omega})$ is the domain bounded by two horocycles internally tangent to $\partial \Delta$ at $z=1$.

To proceed we need the following lemma from [22].

Lemma 6. Let $h \in \Sigma[1]$. Then the angular limit

$$
\angle \lim _{z \rightarrow 1}(1-z) h^{\prime}(z)
$$

exists and is either a positive real number or infinity.

2.2. Main results. We begin with a description of the class $\Sigma_{A}^{0}[1]$. See also [11]. 
Theorem 7. Let $h \in \Sigma[1]$. The following assertions are equivalent:

(i) $h \in \Sigma_{A}^{0}[1]$, i.e.,

$$
\angle \lim _{z \rightarrow 1}(1-z) h^{\prime}(z)=\mu
$$

exists finitely;

(ii) $\Omega=h(\Delta)$ lies in a horizontal strip;

(iii) $h$ is a Bloch function, i.e.,

$$
\|h\|_{B}:=\sup _{z \in \Delta}\left(1-|z|^{2}\right)\left|h^{\prime}(z)\right|<\infty .
$$

Moreover, in this case,

(a) the smallest horizontal strip which contains $h(\Delta)$ is

$$
\left\{w \in \mathbf{C}:|\operatorname{Im} w-a|<\frac{\pi \mu}{2}\right\}
$$

where

$$
a=\lim _{r \rightarrow 1^{-}} \operatorname{Im} h(r) .
$$

(b) $2|\mu| \leq\|h\|_{B} \leq 4|\mu|$.

Remark 8. One of the tools we use in the proof of our results is the Koebe Distortion Theorem (see, for example, [30]) which asserts:

If $h$ is a univalent function on $\Delta$, then

$$
\frac{1}{4}\left(1-|z|^{2}\right)\left|h^{\prime}(z)\right| \leq \delta(h(z)) \leq\left(1-|z|^{2}\right)\left|h^{\prime}(z)\right|, \quad z \in \Delta,
$$

where

$$
\delta(w)=\operatorname{dist}(w, \partial h(\Delta)), w \in h(\Delta) .
$$

Once the equivalence of (i)-(iii) and assertion (a) are proved, we have by (27) the following estimate:

$$
\frac{\pi|\mu|}{2} \leq\|h\|_{B} \leq 2 \pi|\mu|
$$

Clearly, assertion (b) improves (28). Such estimates are very important in finding non-trivial bounds for integral means of $|h(z)|$, as well as its even powers (see, for example, [30], p. 186).

Proof of Theorem 7. The equivalence of assertions (i) and (ii), as well as assertion (a), are proved in [22]. The implication (ii) $\Longrightarrow$ (iii) follows immediately from (27).

Now we will show that (iii) implies (ii). Assume to the contrary that there is no horizontal strip which contains $h(\Delta)$. Then for each $M>0$, there are two points $w_{1}$ and $w_{2}$ in $h(\Delta)$ such that

$$
\operatorname{Im} w_{1}-\operatorname{Im} w_{2}>2 M
$$

Take any continuous curve $\Gamma \subset h(\Delta)$ ending at the points $w_{1}$ and $w_{2}$, and consider the half-strip

$$
\Omega_{1}=\left\{w \in \mathbf{C}: \operatorname{Re} w>b, \operatorname{Im} w_{2}<\operatorname{Im} w<\operatorname{Im} w_{1}\right\},
$$

where $b=\max _{w \in \Gamma} \operatorname{Re} w$. Since $h \in \Sigma(1)$, this half-strip $\Omega_{1}$ must lie in $\Omega=h(\Delta)$. If now $l$ is the midline of $\Omega_{1}$, then one can find $w \in l$ such that $\delta(w)=\operatorname{dist}(w, \partial \Omega)>M$. Since $M$ is arbitrary, this contradicts (iii) by (27). Thus (iii) does indeed imply (ii). 
Finally, it remains to be shown that assertion (b) holds. The left-hand side of the inequality in (b) is immediate. To prove the right-hand side of this inequality, we consider the function $\widehat{h} \in \operatorname{Hol}(\Delta, \mathbf{C})$ defined by $\widehat{h}(z)=\exp \left(-\frac{1}{\mu} h(z)\right)$. It has been shown in [22] that this function is a univalent (starlike with respect to a boundary point) function on $\Delta$ with $h(z) \neq 0, z \in \Delta$. Therefore it follows from Proposition 4.1 in [30] that $g(z):=\log \widehat{h}(z)$ is a Bloch function with

$$
\|g(z)\|_{B} \leq 4 .
$$

But $g(z)=-\frac{1}{\mu} h(z)$. Hence

$$
\|h(z)\|_{B} \leq 4|\mu| .
$$

So, according to relations (2) and (17), we see that a function $h$ belongs to $\Sigma[1]$ if and only if the function $f$ defined by (17) belongs to the class $\mathscr{G}$ [1]. Moreover, $|\operatorname{Im} h(z)| \leq M$ for some $M<\infty$ if and only if $f$ is of hyperbolic type (see [22] and [11]). In our setting this case corresponds to the class $\Sigma_{A}^{\circ}[1]$ (i.e., $\alpha=0$ ).

Now we begin to study the classes $\Sigma_{A}^{\alpha}[1]$, respectively, $\Sigma^{\alpha}[1], \alpha>0$, of functions $h \in \Sigma[1]$, for which

$$
\angle \lim _{z \rightarrow 1}(1-z)^{1+\alpha} h^{\prime}(z)=: \mu .
$$

exists finitely and is different from zero.

Remark 9. Note that these classes arise naturally, if $h(\Delta)$ is contained in a half-plane $\Pi=\left\{w \in \mathbf{C}: w=h_{1}(z):=\frac{i b z}{1-z}, z \in \Delta\right\}(b \neq 0)$ and $\psi=h_{1}^{-1} \circ h$ has a Dini smooth corner of opening $\pi \alpha, 0<\alpha \leq 1$, at the point $z=1$ (see [30], p. 52). Indeed, in this case the limits

$$
A=\lim _{z \rightarrow 1} \frac{1-\psi(z)}{(1-z)^{\alpha}} \quad \text { and } \quad B=\lim _{z \rightarrow 1} \frac{\psi^{\prime}(z)}{(1-z)^{\alpha-1}}
$$

exist finitely and are different from zero. Moreover, it follows from the proof of Theorem 3.9 in [30] that $B=\alpha A$. Thus we have from formula (25) that the limit

$$
\lim _{z \rightarrow 1}(1-z)^{1+\alpha} h^{\prime}(z)=\lim _{z \rightarrow 1} \frac{(1-z)^{\alpha}}{1-\psi(z)} \cdot \lim _{z \rightarrow 1} \frac{(1-z) \psi^{\prime}(z)}{1-\psi(z)} \cdot i b=: \mu
$$

is also finite.

Moreover, in this case formula (26) implies that

$$
\nu:=\lim _{z \rightarrow 1}(1-z)^{\alpha} h(z)=i b \lim _{z \rightarrow 1} \frac{(1-z)^{\alpha} \psi(z)}{1-\psi(z)}=\mu / \alpha
$$

also exists finitely and is different from zero. So, $h(\Delta)$ has a corner of opening $\pi \alpha$, $0<\alpha \leq 1$, at infinity (see [30], p. 54 and Proposition 3.10).

We will see below (see Section 3) that if $h(\Delta)$ contains a half-plane $h_{1}(\Delta)$ and $\varphi=h^{-1} \cdot h_{1}$ has a Dini smooth corner of opening $\pi \gamma$, then $\frac{1}{2} \leq \gamma \leq 1$ and $h \in \Sigma_{A}^{\alpha}[1]$ with $\alpha=\frac{1}{\gamma} \in[1,2]$.

In general, we have the following simple assertion.

Lemma 10. If for some $h \in \Sigma[1]$ there is $\alpha \in \mathbf{R}$ such that $\angle \lim _{z \rightarrow 1}(1-z)^{1+\alpha} h^{\prime}(z)=$ $\mu \neq 0, \infty$, then $\alpha \in[0,2]$. 

0 and

Proof. Denote $p(z):=\frac{1}{(1-z)^{2} h^{\prime}(z)}$. Then by Proposition 3, we have $\operatorname{Re} p(z) \geq$

$$
\angle \lim _{z \rightarrow 1}(1-z)^{1-\alpha} p(z)=\frac{1}{\mu} \neq 0, \infty .
$$

But, it follows by Lemma 6 that the limit

$$
\angle \lim _{z \rightarrow 1}(1-z) p(z) \geq 0
$$

is finite. This contradicts (30) if $\alpha<0$. On the other hand, for any $r \in(0,1)$,

$$
\left|(1-r)^{1-\alpha} p(r)\right| \geq \operatorname{Re} p(0) \cdot \frac{(1-r)^{2-\alpha}}{1+r} .
$$

Therefore, $\alpha \leq 2$.

To study the classes $\Sigma_{A}^{\alpha}[1]$ (respectively, $\Sigma^{\alpha}[1]$ ) for $\alpha>0$, we need the following notion.

- We say that a generator $f$ belongs to the class $\mathscr{G}_{A}^{\alpha}[1]\left(\mathscr{G}^{\alpha}[1]\right), \alpha>0$, if

$$
\begin{aligned}
& \angle \lim _{z \rightarrow 1} \frac{f(z)}{(1-z)^{1+\alpha}}=a \neq 0, \\
& \left(\lim _{\substack{z \rightarrow 1 \\
z \in \Delta}} \frac{f(z)}{(1-z)^{1+\alpha}}=a \neq 0\right) .
\end{aligned}
$$

It is clear that $h \in \Sigma_{A}^{\alpha}[1]$ (respectively, $\Sigma_{A}^{\alpha}[1]$ ) if and only if the function $f$ defined by (17) belongs to the class $\mathscr{G}_{A}^{\alpha}[1]$ (respectively, $\mathscr{G}^{\alpha}[1]$ ) with $a=-\frac{1}{\mu}$. Of course, if $\alpha>0$, then $f$ is of parabolic type.

The following example shows that the class $\Sigma^{\alpha}[1]$ (respectively, $\mathscr{G}^{\alpha}[1]$ ) is a proper subset of the class $\Sigma_{A}^{\alpha}[1]$ (respectively, $\mathscr{G}_{A}^{\alpha}[1]$ ).

Example 11. Consider the function $f \in \operatorname{Hol}(\Delta, \mathbf{C})$ defined by

$$
f(z)=-(1-z)^{2}\left[1-\exp \left(-\frac{1+z}{1-z}\right)\right]^{\beta}(1-z)^{1-\beta}
$$

$0<\beta \leq 1$.

It follows from the Berkson-Porta representation formula that $f \in \mathscr{G}[1]$. Moreover, $f \in \mathscr{G}_{A}^{\alpha}[1], \alpha=2-\beta$, since the angular limit

$$
\angle \lim _{z \rightarrow 1} \frac{f(z)}{(1-z)^{1+\alpha}}=-1 .
$$

At the same time the unrestricted limit

$$
\lim \frac{f(z)}{(1-z)^{1+\alpha}}
$$

does not exist, i.e., $f \notin \mathscr{G}^{\alpha}[1]$.

Theorem 12. Let $h \in \Sigma_{A}^{\alpha}[1]$ with

$$
\angle \lim _{z \rightarrow 1}(1-z)^{1+\alpha} h^{\prime}(z)=: \mu .
$$

The following two assertions hold:

(A) $\alpha \in[0,2]$. 
(B) Assume that for some $z \in \Delta$, the trajectory $\left\{F_{t}(z)\right\}_{t \geq 0}$, where $F_{t}(z)=$ $h^{-1}(h(z)+t)$, converges to $z=1$ nontangentially. Then

(i) $|\arg \mu| \leq \frac{\pi}{2} \min \{\alpha, 2-\alpha\}$. Moreover, if $\alpha \in[0,1]$, then equality here is impossible;

(ii) $\lim _{t \rightarrow \infty} t\left(1-F_{t}(z)\right)^{\alpha}=\frac{\mu}{\alpha}$ for all $z \in \Delta$;

(iii) $\lim _{t \rightarrow \infty} \arg \left(1-F_{t}(z)\right)=\frac{1}{\alpha} \arg \mu$ for all $z \in \Delta$.

To establish Theorem 12, we first need the following lemma.

Lemma 13. Let $p \in \operatorname{Hol}(\Delta, \mathbf{C})$ belong to the Carathéodory class, i.e., $\operatorname{Re} p(z) \geq$ 0 for all $z \in \Delta$. Assume that for some $k \in \mathbf{R}$, the angular limit

$$
\angle \lim _{z \rightarrow 1}(1-z)^{k} p(z)=\gamma
$$

exists finitely and is different from zero. Then

(i) $k \in[-1,1]$;

(ii) $|\arg \gamma| \leq \frac{\pi}{2}(1-|k|)$. limit

Proof. Let $p \in \operatorname{Hol}\left(\Delta, \Pi_{+}\right)$and assume that for some $k \in[-1,1]$, the angular

$$
\angle \lim _{z \rightarrow 1}(1-z)^{k} p(z)=\gamma \neq 0
$$

Define a function $\widehat{p} \in \operatorname{Hol}\left(\Pi_{+}, \Pi_{+}\right)$by

$$
\widehat{p}(w)=p\left(\frac{w-1}{w+1}\right), w \in \Pi_{+} .
$$

Then we have

$$
\gamma=\angle \lim _{w \rightarrow \infty} \widehat{p}(w) \frac{2^{k}}{(w+1)^{k}}=\angle \lim _{w \rightarrow \infty} 2^{k} \frac{\widehat{p}(w)}{w^{k}}
$$

Setting here $w=r \rightarrow \infty$, we obtain that $\frac{\gamma}{2^{k}} \in \overline{\Pi^{+}} \backslash\{0\}$, so

$$
|\arg \gamma| \leq \frac{\pi}{2}
$$

Now fix $\beta \in\left(-\frac{\pi}{2}, \frac{\pi}{2}\right)$ and set $w=r e^{i \beta} \rightarrow \infty$ as $r \rightarrow \infty$ in (33). Then we have

$$
\frac{\gamma}{2^{k}}=\lim _{r \rightarrow \infty} \frac{\widehat{p}\left(r e^{i \beta}\right)}{\left(r e^{i \beta}\right)^{k}}=\lim _{r \rightarrow \infty} \frac{1}{r^{k}} \widehat{p}\left(r e^{i \beta}\right) e^{-i \beta k}
$$

Consequently,

$$
\arg \gamma=\lim _{r \rightarrow \infty}\left(\arg \widehat{p}\left(r e^{i \beta}\right)-k \beta\right) .
$$

At the same time, $\arg \left(\widehat{p}\left(r e^{i \beta}\right)\right) \in\left[-\frac{\pi}{2}, \frac{\pi}{2}\right]$ for all $r>0$ and $\beta \in\left(-\frac{\pi}{2}, \frac{\pi}{2}\right)$. Therefore

$$
\arg \gamma+k \beta=\lim _{r \rightarrow \infty}\left(\widehat{p}\left(r\left(e^{i \beta}\right)\right)\right) \in\left[-\frac{\pi}{2}, \frac{\pi}{2}\right] .
$$

Since $\beta \in\left(-\frac{\pi}{2}, \frac{\pi}{2}\right)$ is arbitrary, we conclude that $|\arg \gamma| \leq \frac{\pi}{2}(1-|k|)$. 
Remark 14. The proof of Lemma 13 presented here is due to Díaz-Madrigal [17]. This lemma as well as Theorem 12 were proved in [23] under the stronger restriction that the unrestricted limits exist. So, Theorem 12 improves upon the results obtained there.

Proof of Theorem 12. Since $f\left(=-\frac{1}{h^{\prime}}\right) \in \mathscr{G}_{A}^{\alpha}[1]$ admits the representation (15), assertion (A) of the theorem is a direct consequence of Lemma 13. Assume now that there is a point $z \in \Delta$ such that the trajectory $\left\{F_{t}(z)\right\}_{t \geq 0}$ converges to the DenjoyWolff point $\tau=1$ nontangentially. For this point $z \in \Delta$ we denote $u(t):=F_{t}(z)$, $t \geq 0$. Then it follows from the Cauchy problem (18) that

$$
\frac{d u}{(1-u)^{1+\alpha}}=-\frac{f(u)}{(1-u)^{1+\alpha}} d t, \quad u(0)=z,
$$

which is equivalent to the integral equation

$$
\frac{1}{t} \cdot \frac{1}{(1-u(t))^{\alpha}}=-\frac{\alpha}{t} \int_{0}^{t} \frac{f(u(s)) d s}{(1-u(s))^{1+\alpha}}+\frac{1}{t} \cdot \frac{1}{(1-z)^{\alpha}} .
$$

Now it follows from (31) that

and

$$
\lim _{t \rightarrow \infty} t(1-u(t))^{\alpha}=-\frac{1}{\alpha a}=\frac{\mu}{\alpha}
$$

$$
\lim _{t \rightarrow \infty} \arg (1-u(t))=-\frac{1}{\alpha} \arg (-a)=\frac{1}{\alpha} \arg \mu .
$$

This means that the trajectory $\left\{F_{t}(z)\right\}_{t \geq 0}$ has an asymptote at its attractive point $\tau=1$. Next, it follows from Theorem 2.9 in [11] that for each $z \in \Delta$, the trajectory $\left\{F_{t}(z)\right\}_{t \geq 0}$ has the same asymptote and all the trajectories converge to $\tau=1$ nontangentially.

Repeating the above considerations for an arbitrary $z \in \Delta$, we arrive at assertions (ii) and (iii) of the theorem.

Applying again Lemma 13 and (15), we obtain

$$
|\arg (-a)| \leq \frac{\pi}{2} \min \{\alpha, 2-\alpha\}
$$

But if $\alpha \in(0,1]$, then equality here is impossible, since otherwise we see by (iii) that

$$
\lim _{t \rightarrow \infty} \arg \left(1-F_{t}(z)\right)= \pm \frac{\pi}{2},
$$

which contradicts the nontangential convergence. This proves assertion (i) and we are done.

Remark 15. We note that the conclusion of Theorem 12 remains, of course, valid if we replace condition (31) by (32). Moreover, the requirement of nontangential convergence in this case is not necessary. In addition, formulas (i) and (iii) show that if $\alpha>1(\alpha \leq 2)$, then all the trajectories must necessarily converge in a nontangential way.

This proves the following assertion.

Corollary 16. Let $f \in \mathscr{G}^{\alpha}[1]$. The following assertions are equivalent:

(i) for some $z \in \Delta$, the trajectory $\left\{F_{t}(z)\right\}_{t \geq 0}$ converges to $z=1$ tangentially; 
(ii) for all $z \in \Delta$, the trajectories $\left\{F_{t}(z)\right\}_{t \geq 0}$ converge to $z=1$ tangentially;

(iii) $|\arg (-a)|=\frac{\pi}{2} \alpha$.

Moreover, in this case $0<\alpha \leq 1$.

Remark 17. Note that the nontangential convergence of the trajectory $\left\{F_{t}(z)\right\}_{t \geq 0}$ to the Denjoy-Wolff point $z=1$ of the semigroup $S$ means that

$$
\inf _{t \geq 0} \frac{1-\left|F_{t}(z)\right|}{\left|1-F_{t}(z)\right|}>0
$$

We will see that this condition implies that there is no horizontal half-plane which contains $h(\Delta)$.

In the opposite direction, the condition

$$
\lim _{t \geq 0} \frac{1-\left|F_{t}(z)\right|}{\left|1-F_{t}(z)\right|}=0
$$

implies that the trajectory $\left\{F_{t}(z)\right\}_{t \geq 0}$ converges tangentially to $z=1$ and conversely. So, the question is whether the latter condition is sufficient to ensure that $h(\Delta)$ lies in a horizontal half-plane.

The following theorem shows that for $h \in \Sigma^{\alpha}[1]$ the existence of a horizontal half-plane containing $h(\Delta)$ is, in fact, equivalent to the stronger condition that the ratio $\frac{1-\left|F_{t}(z)\right|}{\left|1-F_{t}(z)\right|}$ converges to zero faster than $\frac{1}{t}$. Moreover, for $\alpha=1$, we will see that the limit

$$
\lim _{t \rightarrow \infty} \frac{t\left(1-\left|F_{t}(z)\right|\right)}{\left|1-F_{t}(z)\right|}=L(z)
$$

exists and is different from zero if and only if all the trajectories converge to $z=1$ strongly tangentially, i.e., for each $z \in \Delta$ there is a horocycle internally tangent to $\partial \Delta$ at the point $z=1$ which does not contain $\left\{F_{t}(z)\right\}_{t>s}$ for some $s \geq 0$.

We recall that by $\Sigma^{\alpha}[1], \alpha \in[0,2]$, we denote the subclass of $\Sigma[1]$ which consists of those functions $h$ for which the unrestricted limit $\lim _{z \rightarrow 1}(1-z)^{1+\alpha} h^{\prime}(z)$ exists finitely and is different from zero.

Theorem 18. Let $h \in \Sigma^{\alpha}[1]$ with $\alpha \in(0,2]$,

$$
\mu:=\lim _{\substack{z \rightarrow 1 \\ z \in \Delta}}(1-z)^{1+\alpha} h^{\prime}(z) \neq 0, \infty
$$

and let $h^{-1}(h(z)+t)=: F_{t}(z)$. Then $h(\Delta)$ lies in a horizontal half-plane if and only if

$$
\sup _{t \geq 0} \frac{t\left(1-\left|F_{t}(z)\right|\right)}{\left|1-F_{t}(z)\right|}=: M(z)<\infty .
$$

Moreover, in this case the following assertions hold:

(a) $\alpha$ must belong to the half-open interval $(0,1]$;

(b) $|\arg \mu|=\frac{\pi}{2} \alpha$;

(c) all the trajectories $\left\{F_{t}(z)\right\}, t \geq 0, z \in \Delta$, converge tangentially to the point $z=1$ 
(d) if $\Pi=\left\{w=\frac{i b z}{1-z}: z \in \Delta\right\}, b \in \mathbf{R} \backslash\{0\}$, is a half-plane containing $h(\Delta)$, then

$$
b \cdot \arg \mu>0 .
$$

In other words, $\operatorname{Im} h(z)$ is bounded from below if and only if $\arg \mu>0$. Otherwise $(\arg \mu<0)$, the set $\{\operatorname{Im} h(z): z \in \Delta\}$ is bounded from above. Recall that since $\alpha \neq 0$, $\operatorname{Im} h(z)$ cannot be bounded from both above and below.

Corollary 19. Let $h \in \Sigma^{\alpha}[1]$ with $\alpha \in(0,2]$ and

$$
\mu:=\lim _{\substack{z \rightarrow 1 \\ z \in \Delta}}(1-z)^{1+\alpha} h^{\prime}(z) \neq 0, \infty .
$$

If either $\alpha>1$ or $|\arg \mu| \neq \frac{\pi}{2} \alpha$, then there is no horizontal half-plane which contains $h(\Delta)$. In particular, if $\mu$ is real, then

$$
\sup _{z \in \Delta} \operatorname{Im} h(z)=\infty \quad \text { and } \quad \inf _{z \in \Delta} \operatorname{Im} h(z)=-\infty .
$$

Example 20. Consider the function $h \in \operatorname{Hol}(\Delta, \mathbf{C})$ defined by

$$
h(z)=\frac{\mu}{K+1}\left[\frac{1}{(1-z)^{K+1}}-1\right], \quad z \in \Delta,
$$

with $K \neq-1, K \in \mathbf{R}$ and $\mu \in \mathbf{C} \backslash\{0\}$. Since $h(0)=0$ and

$$
h^{\prime}(z)=\frac{\mu}{(1-z)^{2+K}}
$$

we have

$$
\operatorname{Re}\left[(1-z)^{2} h^{\prime}(z)\right]=\operatorname{Re}\left[\mu \frac{1}{(1-z)^{K}}\right] \geq 0
$$

if and only if $-1<K \leq 1$ and $|\arg \mu| \leq \frac{\pi}{2}-\frac{\pi K}{2}$.

We also see that

$$
\angle \lim _{z \rightarrow 1}(1-z)^{1+\alpha} \cdot h^{\prime}(z)=\mu \lim _{z \rightarrow 1}(1-z)^{1+\alpha} \cdot \frac{1}{(1-z)^{K+2}}
$$

is finite and different from zero (and equal to $\mu$ ) if and only if $\alpha=K+1$. Thus $\alpha$ must lie in $(0,2]$. If $K>0$ or $|\arg \mu|<\frac{\pi}{2}-\frac{\pi K}{2}$, then it follows from Theorem 18 and Corollary 19 that there is no horizontal half-plane which contains $h(\Delta)$.

To prove Theorem 18 we use again the relationship between the class $\Sigma[1]$ of functions convex in the positive direction of the real axis and the class $\mathscr{G}[1]$ of semigroup generators established in formulas (2) and (17).

Proof of Theorem 18. For a domain $\Omega \subset \mathbf{C}$, let $\delta(w)=\operatorname{dist}(w, \partial \Omega), w \in \Omega$. Since $h \in \Sigma^{\alpha}[1] \subset \Sigma[1]$, the fact that $\Omega=h(\Delta)$ lies in a half-plane can be described as follows: $\lim _{t \rightarrow \infty} \delta(h(z)+t)=: k(z)$ is finite for each $z \in \Delta$ (see [11]).

Using again the Koebe Distortion Theorem, $\delta(h(\zeta)) \leq\left(1-|\zeta|^{2}\right)\left|h^{\prime}(\zeta)\right| \leq 4 \delta(h(\zeta))$, setting $\zeta=F_{t}(z)$, and using the equality $h\left(F_{t}(z)\right)=h(z)+t$, we get

$$
\delta(h(z)+t) \leq\left(1-\left|F_{t}(z)\right|^{2}\right)\left|h^{\prime}\left(F_{t}(z)\right)\right| \leq 4 \delta(h(z)+t)
$$

or

$$
\delta(h(z)+t) \leq \frac{t\left(1-\left|F_{t}(z)\right|^{2}\right)}{\left|1-F_{t}(z)\right|}\left|1-F_{t}(z)\right|^{1+\alpha}\left|h^{\prime}\left(F_{t}(z)\right)\right| \frac{1}{t\left|1-F_{t}(z)\right|^{\alpha}} \leq 4 \delta(h(z)+t) .
$$


Since $h \in \Sigma^{\alpha}[1]$, we see that for each $z \in \Delta$,

$$
\lim _{t \rightarrow \infty}\left|1-F_{t}(z)\right|^{1+\alpha}\left|h^{\prime}\left(F_{t}(z)\right)\right|=|\mu| .
$$

In addition, we know that in this case the generator $f$ of the semigroup $h^{-1}(h(z)+$ $t)=: F_{t}(z)$ belongs to $\mathscr{G}^{\alpha}[1]$. Thus it follows from assertion (ii) of Theorem 12 and the remark following its proof that $\lim _{t \rightarrow \infty} t\left|1-F_{t}(z)\right|^{\alpha}=\frac{|\mu|}{\alpha}$ for all $z \in \Delta$. Now recall that

$$
a:=\lim _{\substack{z \rightarrow 1 \\ z \in \Delta}} \frac{f(z)}{(1-z)^{1+\alpha}}=\frac{-1}{\mu} .
$$

So the function $k(z)$ is finite if and only if for each $z \in \Delta$,

$$
\sup _{t \geq 0} \frac{t\left(1-\left|F_{t}(z)\right|\right)}{\left|1-F_{t}(z)\right|}=: M(z)<\infty .
$$

This condition, in its turn, implies that

$$
\lim _{t \rightarrow \infty} \frac{1-\left|F_{t}(z)\right|}{\left|1-F_{t}(z)\right|}=0
$$

which means that all the trajectories $\left\{F_{t}(z)\right\}_{t \geq 0}$ converge tangentially to the point $z=1$ as $t \rightarrow \infty$. Then, by Theorem 12 (iii), $|\arg \mu|=\frac{\pi}{2} \alpha$. In addition, inequality (i) in Theorem 12 shows that if $\alpha>1$, then $|\arg \mu|=\frac{\pi}{2} \alpha \leq \frac{\pi}{2}(2-\alpha)$, which is impossible.

To prove (d), our last assertion, we consider the function $\psi: \Delta \mapsto \Delta$ defined by $\psi(z)=h_{1}^{-1}(h(z))$, where $h_{1}(z)=\frac{i b z}{1-z}$. Then we have by Proposition 4 (see formulas (21), (22))

$$
\begin{aligned}
\lim _{t \rightarrow \infty} \frac{1-\psi\left(F_{t}(z)\right)}{\left(1-F_{t}(z)\right)^{\alpha}} & =\lim _{t \rightarrow \infty} \frac{t\left(1-G_{t}(\psi(z))\right.}{t\left(1-F_{t}(z)\right)^{\alpha}} \\
& =\frac{\alpha}{\mu} \lim _{t \rightarrow \infty} t\left(1-\frac{i b \psi(z)+t(1-\psi(z))}{i b+t(1-\psi(z))}\right)=\frac{\alpha}{\mu} \cdot i b .
\end{aligned}
$$

If $\alpha<1$, then the function $\frac{1-\psi(z)}{(1-z)^{\alpha}}$ does not admit negative real values. Then it follows from the generalized Lindelöf Theorem (see Theorem 2.20 in [10], p. 42) that

$$
\gamma=\angle \lim _{z \rightarrow 1} \frac{1-\psi(z)}{(1-z)^{\alpha}}=\frac{\alpha}{\mu} \cdot i b .
$$

If $\alpha=1$, then this limit also exists by the Julia-Wolff-Carathéodory Theorem (see, for example, [38] and [37]). Note that in this case $\mu$ is pure imaginary; hence the limit $\gamma$ in (34) is a real number.

In addition, since the function $1-\psi(z)$ is of positive real part, we get by Lemma 13 that

$$
|\arg \gamma| \leq \frac{\pi}{2}(1-\alpha)
$$


Then, by (34) and (35), we clearly have that $b>0$ if and only if $\arg \mu>0$. Noting that by assertion (ii), $|\arg \mu|=\frac{\pi}{2} \alpha$ and $\alpha>0$, we conclude that $\mu$ cannot be a real number and Theorem 18 as well as Corollary 19 are proved.

Now, using Proposition 4, we get the following assertion.

Theorem 21. Let $S=\left\{F_{t}\right\}_{t>0}$ be a semigroup of parabolic self-mappings of $\Delta$ generated by $f \in \mathscr{G}^{\alpha}[1]$, i.e.,

$$
\lim _{z \rightarrow 1} \frac{f(z)}{(1-z)^{1+\alpha}}=a
$$

exists finitely and is different from zero. The following two assertions are equivalent:

(i) $\left\{F_{t}\right\}_{t \geq 0}$ outer conjugates with a group $\left\{G_{t}\right\}_{t \in \mathbf{R}}$ of parabolic automorphisms of $\Delta$ having 1 as the Denjoy-Wolff point, i.e., there is a conformal self-mapping $\psi: \Delta \mapsto \Delta$ such that

$$
\psi\left(F_{t}(z)\right)=G_{t}(\psi(z)), \quad t \geq 0, \quad z \in \Delta
$$

(ii)

$$
\sup _{t \geq 0} \frac{t\left(1-\left|F_{t}(z)\right|\right)}{\left|1-F_{t}(z)\right|}=: M(z)<\infty, \quad z \in \Delta .
$$

Moreover, in this case

(a) $\alpha \in(0,1]$;

(b) $|\arg (-a)|=\frac{\pi}{2} \alpha$;

(c) all the trajectories $\left\{F_{t}(z)\right\}_{t \geq 0}, z \in \Delta, t \geq 0$, converge tangentially to the point $z=1$.

As we have already mentioned, when $\alpha=1$ condition (ii) is equivalent to the so-called strongly tangential convergence (see Definition 22 below); hence it implies the existence of the conjugating function.

Moreover, it turns out that strongly tangential convergence is possible if and only if $\alpha=1$. To see this, we denote by $d(z)$ the non-euclidean distance from a point $z \in \Delta$ to the boundary point $\zeta=1$ :

$$
d(z):=\frac{|1-z|^{2}}{1-|z|^{2}}
$$

The sets $\{z \in \Delta: d(z)<k\}, k>0$, are horocycles internally tangent to $\partial \Delta$ at $z=1$. It follows from the Julia-Wolff-Carathéodory theorem for semigroups (see [20] and [38]) that for each $z \in \Delta$, the function $d\left(F_{t}(z)\right)$ is decreasing, so the limit

$$
\lim _{t \rightarrow \infty} d\left(F_{t}(z)\right)=\varepsilon(z)
$$

exists and is nonnegative. It can be shown that either $\varepsilon(z)>0$ for all $z \in \Delta$ or $\varepsilon(z)=0$ identically (see, for example, [40]).

Definition 22. We say that a semigroup $S=\left\{F_{t}\right\}_{t \geq 0}$ is strongly tangentially convergent if $\varepsilon(z)>0$.

Geometrically, this means that for each $z \in \Delta$, there is a horocycle $E$ internally tangent to $\partial \Delta$ at $z=1$ such that the trajectory $\left\{F_{t}(z)\right\}_{t \geq 0}$ lies outside $E$. Sometimes a strongly tangentially convergent semigroup is said to be of finite shift (see [14]). 
Theorem 23. Let $h \in \Sigma^{\alpha}[1], \alpha \in[0,2]$, and let $S=\left\{F_{t}\right\}_{t \geq 0}$ be defined by $F_{t}(z)=h^{-1}(h(z)+t), z \in \Delta, t \geq 0$. The following two assertions hold.

(A) Assume that for some $z \in \Delta$, the trajectory $\left\{F_{t}(z)\right\}_{t \geq 0}$ converges to $z=1$ strongly tangentially. Then

(i) $\alpha=1$;

(ii) $h(\Delta)$ is contained in a half-plane.

(B) Conversely, assume that conditions (i) and (ii) are fulfilled. Then all the trajectories $\left\{F_{t}(z)\right\}_{t \geq 0}, z \in \Delta$, converge to $z=1$ strongly tangentially.

Moreover,

$$
\mu=\lim _{z \rightarrow 1}(1-z)^{2} h^{\prime}(z)
$$

is purely imaginary, and either

or

$$
\operatorname{Im} h(z)>\frac{1}{2 \varepsilon(0) \cdot \operatorname{Im} \frac{1}{\mu}}, z \in \Delta, \quad \text { if } \operatorname{Im} \mu>0,
$$

$$
\operatorname{Im} h(z)<\frac{1}{2 \varepsilon(0) \cdot \operatorname{Im} \frac{1}{\mu}}, z \in \Delta, \quad \text { if } \operatorname{Im} \mu<0 .
$$

Proof. As we have already mentioned, if the trajectory $\left\{F_{t}(z)\right\}_{t \geq 0}$ converges to $z=1$ (strongly) tangentially, then $0<\alpha \leq 1$. We claim that $\alpha$ cannot be less than 1. Indeed, consider

$$
d\left(F_{t}(z)\right):=\frac{\left|1-F_{t}(z)\right|^{2}}{1-\left|F_{t}(z)\right|^{2}} .
$$

Since $h \in \Sigma^{\alpha}[1]$, we have that for $t$ large enough,

$$
\left|1-F_{t}(z)\right|^{1+\alpha} \mid h^{\prime}\left(F_{t}(z)|\leq| \mu \mid+1\right. \text {. }
$$

Then again by the Koebe Distortion Theorem we get

$$
d F_{t}(z)=\frac{\left|1-F_{t}(z)\right|^{1+\alpha}}{\left(1-\left|F_{t}(z)\right|^{2}\right)} \frac{\left|h^{\prime}\left(F_{t}(z)\right)\right|}{\left|h^{\prime}\left(F_{t}(z)\right)\right|} \cdot\left|1-F_{t}(z)\right|^{1-\alpha} \leq \frac{|\mu|+1}{\delta(h(z)+t)}\left|1-F_{t}(z)\right|^{1-\alpha} .
$$

Thus, if at least one of conditions (i) or (ii) does not hold, i.e., $\alpha<1$ or $\lim _{t \rightarrow \infty} \delta(h(z)$ $+t)=\infty$, then $d\left(F_{t}(z)\right)$ must converge to zero, and this is a contradiction. Assertion (A) is proved.

Now we prove the converse assertion (B). To this end, assume that (i) and (ii) hold. Fix any $w \in h(\Delta)$ and denote by $\delta(w)$ the euclidean distance from $w$ to $\partial h(\Delta)$. Then condition (ii) of the theorem can be rewritten as follows:

$$
\lim _{t \rightarrow \infty} \delta(h(z)+t)=k(z), \quad z \in \Delta,
$$

is finite. Note that condition (36) and Theorem 2.9 (b) in [11] imply that all trajectories converge to the point $\tau=1$ tangentially. To prove strongly tangential convergence, we use again the Koebe Distortion Theorem which asserts that for all $w \in h(\Delta)$,

$$
\delta(w) \leq\left(1-|\zeta|^{2}\right)\left|h^{\prime}(\zeta)\right| \leq 4 \delta(w),
$$

where $w=h(\zeta), \zeta \in \Delta$.

Setting $\zeta=F_{t}(z)$ in (37) and using the equality $h\left(F_{t}(z)\right)=h(z)+t$, we have

$$
\delta(h(z)+t) \leq\left(1-\left|F_{t}(z)\right|^{2}\right) h^{\prime}\left(F_{t}(z)\right) \leq 4 \delta(h(z)+t)
$$


or

$$
\delta(h(z)+t) \leq \frac{\left|1-F_{t}(z)\right|^{2}\left|h^{\prime}\left(F_{t}(z)\right)\right|}{d\left(F_{t}(z)\right)} \leq 4 \delta(h(z)+t)
$$

where $d\left(F_{t}(z)\right)=\frac{\left|1-F_{t}(z)\right|^{2}}{1-\left|F_{t}(z)\right|^{2}}$ converges to $\varepsilon(z) \geq 0$ as $t \rightarrow \infty$. Since

$$
\lim _{t \rightarrow \infty}\left|1-F_{t}(z)\right|^{2}\left|h^{\prime}\left(F_{t}(z)\right)\right|=|\mu|,
$$

we get the inequality

$$
k(z) \leq \frac{|\mu|}{\varepsilon(z)} \leq 4 k(z),
$$

which shows that $k(z)$ is finite if and only if $\varepsilon(z)>0$. This proves the implication $(\mathrm{B}) \Longrightarrow(\mathrm{A})$.

To prove our last assertion, we use Theorem 3 from [40] which asserts that if $f \in \mathscr{G}^{1}[1]$ with $f^{\prime \prime}(1) \neq 0$, then the trajectories $\left\{F_{t}(z)\right\}_{t \geq 0}, z \in \Delta$, converge to $\tau=1$ strongly tangentially if and only if the net

$$
g_{t}(z)=d_{t}(0)\left[\frac{1+F_{t}(z)}{1-F_{t}(z)}-\frac{2 i \operatorname{Im} \overline{F_{t}(0)}}{\left|1-F_{t}(0)\right|^{2}}\right]
$$

converges locally uniformly as $t \rightarrow \infty$ to a holomorphic function

$$
g: \Delta \mapsto \Pi_{+}=\{w \in \mathbf{C}: \operatorname{Re} w \geq 0\},
$$

which solves Abel's equation

$$
g\left(F_{t}(z)\right)=g(z)+i b t
$$

where $b=-\varepsilon(0) \operatorname{Im} f^{\prime \prime}(1)$. It was also shown there that the condition $\varepsilon(0)>0$ implies that $a=\frac{1}{2} f^{\prime \prime}(1)$ (hence $\mu=-\frac{1}{a}$ ) is purely imaginary.

Differentiating now (39) at $t=0^{+}$, we get

$$
g^{\prime}(z)=-\frac{i b}{f(z)} .
$$

In addition, (38) implies that $\operatorname{Re} g(z) \geq 0$ and $g(0)=1$. But it now follows from (17) that

$$
g(z)=i b h(z)+1
$$

or

$$
h(z)=\frac{i}{\varepsilon(0) \operatorname{Im} f^{\prime \prime}(1)}[g(z)-1]=\frac{i}{2 \varepsilon(0)\left(-\operatorname{Im} \frac{1}{\mu}\right)}[g(z)-1] .
$$

So, $\operatorname{Im} h(z)>\frac{1}{2 \varepsilon(0) \cdot \operatorname{Im} \frac{1}{\mu}}$ if $\operatorname{Im} \mu>0$, and $\operatorname{Im} h(z)<\frac{1}{2 \varepsilon(0) \cdot \operatorname{Im} \frac{1}{\mu}}$ if $\operatorname{Im} \mu<0$. Theorem 23 is proved.

Alternatively, the implication $(\mathrm{B}) \Longrightarrow(\mathrm{A})$ can also be proved by using the following Proposition and Theorem 18. 
Proposition 24. Let $S=\left\{F_{t}\right\}_{t>0}$ be a semigroup of parabolic type generated by $f \in \mathscr{G}^{1}[1]$, i.e.,

$$
\lim _{z \rightarrow 1} \frac{f(z)}{(1-z)^{2}}=: \frac{1}{2} f^{\prime \prime}(1)=a
$$

exists finitely and is different from zero. Then $\left\{F_{t}\right\}_{t \geq 0}$ converges to $z=1$ strongly tangentially if and only if

$$
\lim _{t \rightarrow \infty} \frac{t\left(1-\left|F_{t}(z)\right|\right)}{\left|1-F_{t}(z)\right|}=L(z)
$$

is finite.

Proof. We already know that

$$
\lim _{t \rightarrow \infty} t\left(1-F_{t}(z)\right)=-\frac{1}{a} .
$$

Therefore we have

$$
\begin{aligned}
\varepsilon(z) & =\lim _{t \rightarrow \infty} \frac{\left|1-F_{t}(z)\right|^{2}}{1-\left|F_{t}(z)\right|^{2}}=\frac{1}{2} \lim _{t \rightarrow \infty} \frac{t\left|1-F_{t}(z)\right| \cdot\left|1-F_{t}(z)\right|}{t\left(1-\left|F_{t}(z)\right|\right)} \\
& =\frac{1}{2|a|} \lim _{t \rightarrow \infty} \frac{\left|1-F_{t}(z)\right|}{t\left(1-\left|F_{t}(z)\right|\right)}=\frac{1}{2|a| L(z)} .
\end{aligned}
$$

Thus we see that $\varepsilon(z)>0$ if and only if $L(z)<\infty$.

Using this theorem and Theorem 18, one can easily construct an example of a semigroup which converges tangentially, but not strongly tangentially, to its DenjoyWolff point.

Example 25. Consider a function $h: \Delta \mapsto \mathbf{C}$ conformally mapping the open unit disk $\Delta$ onto the quadrant $\left\{(x, y) \in \mathbf{R}^{2}:-1 \leq x<\infty,-1 \leq y<\infty\right\}$ given by the formula

$$
h(z)=e^{i \frac{\pi}{4}}\left[\sqrt{\frac{1+z}{1-z}}-1\right] .
$$

It is clear that $h \in \Sigma^{\alpha}[1]$, with $\alpha=\frac{1}{2}$ and

$$
\mu=\lim (1-z)^{\alpha+1} h^{\prime}(z)=e^{i \frac{\pi}{4}} .
$$

The corresponding generator $f\left(=-\frac{1}{h^{\prime}}\right)$ is defined by $f(z)=-(1-z)^{2} \sqrt{\frac{1+z}{1-z}} e^{-\frac{\pi}{4} i}$.

Since $h(\Delta)$ lies in a horizontal half-plane, all the trajectories $F_{t}(z)$ must converge to $z=1$ tangentially:

$$
\lim _{t \rightarrow \infty} \arg \left(1-F_{t}(z)\right)= \pm \frac{\arg \mu}{\alpha}= \pm \frac{\pi}{2} .
$$

At the same time no trajectory can converge to $z=1$ strongly tangentially because $\alpha \neq 1$.

Combining this theorem with a result in [41], we get the following rigidity assertion.

Corollary 26. Let $f \in \mathscr{G}^{\alpha}[1]$ for some $\alpha \in[0,2]$ admit the representation

$$
f(z)=a(1-z)^{\alpha+1}+b(1-z)^{3}+\gamma(z),
$$


where

$$
\angle \lim _{z \rightarrow 1} \frac{\gamma(z)}{(1-z)^{3}}=0 .
$$

Assume that the semigroup $S=\left\{F_{t}\right\}_{t \geq 0}$ converges to $z=1$ strongly tangentially. Then

(i) $\alpha=1$; hence $\operatorname{Re} a=0$;

(ii) $\operatorname{Im} b=0$ and $\operatorname{Re} b \leq 0$;

(iii) $\operatorname{Re} b=0$ if and only if $\gamma \equiv 0$, i.e., $f$ is the generator of a group of parabolic automorphisms of $\Delta$.

Under some additional conditions of regularity one can establish a more general criterion for $h(\Delta)$ to lie in a half-plane (not necessarily horizontal).

Let us assume that $h(\Delta)$ is a Jordan domain (the region interior to a Jordan curve). Then the Carathéodory Extension Theorem (see, for example, [30]) asserts that $h$ extends to a homeomorphism of $\bar{\Delta}$ and formula (2) then yields an extension of $F_{t}$ to a homeomorphism of $\bar{\Delta}$ for each $t>0$. We also assume that $h(z)+1 \in h(\Delta)$ for all $z \in \bar{\Delta}$, which implies that $\overline{F_{1}(\Delta)} \subset \bar{\Delta}$.

Finally, we suppose that the generator $f: \Delta \mapsto \mathbf{C}$ of the semigroup $S=\left\{F_{t}\right\}_{t \geq 0}$ $\left(f(z)=-1 / h^{\prime}(z)\right)$ admits the representation

$$
f(z)=a(z-1)^{2}+b(z-1)^{3}+\gamma(z),
$$

where

$$
\lim _{z \rightarrow 1} \frac{\gamma(z)}{(z-1)^{3+\varepsilon}}=0
$$

for some $\varepsilon>0$. In this case one can show (see, for example, [19]) that the single mapping $F_{1}$ can be represented in the form

$$
F_{1}(z)=C^{-1}\left(w+a_{1}+\frac{b_{1}}{w+1}+\Gamma(w+1)\right)
$$

where $w=C(z):=\frac{1+z}{1-z}, a_{1}=F_{1}^{\prime \prime}(1), b_{1}=\left(F_{1}^{\prime \prime}(1)\right)^{2}-\frac{2}{3} F_{1}^{\prime \prime \prime}(1)$ (the Schwarz derivative), and

$$
|\Gamma(w+1)| \leq \frac{C}{|w+1|^{1+\varepsilon}}
$$

It was shown in [5] (p. 62) that in this case there is a (unique) function $g: \Delta \mapsto \mathbf{C}$ with $g(0)=0$ which solves Abel's equation

$$
g\left(F_{1}(z)\right)=g(z)+a_{1} .
$$

Moreover, $\operatorname{Re} g(z)$ is bounded from below if and only if

$$
\operatorname{Re} \overline{a_{1}} \cdot b_{1} \leq 0 \text {. }
$$

Using now Theorem 1 in [19], we obtain

$$
F_{1}^{\prime \prime}(1)=-f^{\prime \prime}(1)
$$

and

So, we see that

$$
F_{1}^{\prime \prime \prime}(1) \equiv \frac{3}{2}\left(f^{\prime \prime}(1)\right)^{2}-f^{\prime \prime \prime}(1) .
$$

$$
a_{1}=F_{1}^{\prime \prime}(1)=-f^{\prime \prime}(1)=-2 a
$$


and

$$
\begin{aligned}
b_{1} & =\left(F_{1}^{\prime \prime}(1)\right)^{2}-\frac{2}{3} F_{1}^{\prime \prime \prime}(1)=\left(F_{1}^{\prime \prime}(1)\right)^{2}-\frac{2}{3}\left[\frac{3}{2}\left(f^{\prime \prime}(1)\right)^{2}-f^{\prime \prime \prime}(1)\right] \\
& =\frac{2}{3} f^{\prime \prime \prime}(1)=\frac{2}{3} 6 b=4 b .
\end{aligned}
$$

Thus condition (40) can be replaced by $\operatorname{Re} \bar{a} b \leq 0$. Since $h(z)=\frac{1}{a} g(z)$, we get the following assertion.

Theorem 27. Let $h \in \Sigma^{1}[1]$ be such that

(a) $h(\Delta)$ is a Jordan domain;

(b) $h(z)+1$ belongs to $h(\Delta)$ for all $z \in \bar{\Delta}$;

(c) $f(z)=-\frac{1}{h^{\prime}(z)}$ admits the representation

$$
f(z)=a(z-1)^{2}+b(z-1)^{3}+\gamma(z),
$$

where

$$
\lim _{z \rightarrow 1} \frac{\gamma(z)}{(z-1)^{3+\varepsilon}}=0
$$

for some $\varepsilon>0$.

Then $h(\Delta)$ lies in a half-plane if and only if

$$
\operatorname{Re} \bar{a} b \leq 0 .
$$

Remark 28. It follows immediately from the Berkson-Porta representation formula that $\operatorname{Re} a \leq 0$. Moreover, under our assumption $\operatorname{Re} a=0$ if and only if the trajectories $\left\{F_{t}(z)\right\}_{t \geq 0}$ converge to $z=1$ strongly tangentially. In addition, since

$$
b=\lim _{z \rightarrow 1} \frac{q(z)}{1-z}
$$

where

$$
q(z)=p(z)+a=-\frac{f(z)}{(1-z)^{2}}+a
$$

is a function with a nonnegative real part, we see that $\operatorname{Re} a=0$ implies that $b$ is a real number, so condition (41) holds automatically.

Finally, we note that it was proved in [40] that if $\operatorname{Re} a=b=0$, then, in fact, $S=\left\{F_{t}\right\}_{t \geq 0}$ consists of parabolic automorphisms of $\Delta$. In this case $h \in \Sigma^{1}[1]$ must be of the form

for some real $k \neq 0$.

$$
h(z)=\frac{i k z}{1-z}
$$

\section{Covering theorems (inner conjugation)}

3.1. Backward flow invariant domains. Let $f \in \mathscr{G}[1]$ be the generator of a semigroup $S=\left\{F_{t}\right\}_{t \geq 0}$ having the Denjoy-Wolff point $\tau=1$, i.e.,

$$
f(1)\left(:=\angle \lim _{z \rightarrow 1} f(z)\right)=0
$$

and

$$
f^{\prime}(1)\left(=\angle \lim _{z \rightarrow 1} \frac{f(z)}{z-1}\right) \geq 0
$$


Definition 29. (See [24]) We say that a simply connected domain $\Omega \subset \Delta$ is a backward flow invariant domain (BFID) for $S$ if $S$ can be extended to a group on $\Omega$.

Since $\Omega$ (if it exists) is simply connected, one can find a Riemann conformal mapping $\varphi: \Delta \mapsto \Delta$ such that $\varphi(\Delta)=\Omega$. It is clear that the family $\left\{G_{t}\right\}_{t \in \mathbf{R}}$ defined by

$$
G_{t}(z)=\varphi^{-1}\left(F_{t}(\varphi(z))\right), \quad t \in \mathbf{R},
$$

forms a group of automorphisms of $\Delta$. Thus, the existence of a BFID for $S$ is equivalent to the existence of an inner conjugator $\varphi: \Delta \mapsto \Delta$,

$$
F_{t}(\varphi(z))=\varphi\left(G_{t}(z)\right), \quad t \geq 0,
$$

where $\left\{G_{t}\right\}_{t \in \mathbf{R}}$ is a group on $\Delta$. Furthermore, since $\tau=1$ is the Denjoy-Wolff point of $S$ it follows from (43), that

$$
\lim _{t \rightarrow \infty} \varphi\left(G_{t}(z)\right)=1
$$

Hence, $\tau=1 \in \bar{\Omega}$.

It is also clear that the group $\left\{G_{t}\right\}$ is not elliptic. Indeed, otherwise there would be a point $\eta \in \Delta$ such that $G_{t}(\eta)=\eta$ for all $t \in \mathbf{R}$. Then (2) would imply that $\varphi(\eta) \in \Delta$ is a fixed point of $S: \varphi(\eta)=\varphi\left(G_{t}(\eta)\right)=F_{t}(\varphi(\eta))$. This would contradict our assumption that $S$ has a boundary Denjoy-Wolff point. Thus the group $\left\{G_{t}\right\}_{t \in \mathbf{R}}$ is either of parabolic or hyperbolic type.

Definition 30. We say that $\Omega$ is of $p$-type if $\left\{G_{t}\right\}_{t \in \mathbf{R}}$ is parabolic and that it is of $h$-type if this group is hyperbolic.

Remark 31. We will see below that if $\Omega$ is of $p$-type, then $S=\left\{F_{t}\right\}_{t \geq 0}$ must also be of parabolic type, i.e., $f^{\prime}(1)=0$. At the same time, if $\Omega$ is of $h$-type then the semigroup $S$ might be of hyperbolic as well as parabolic type (see also Remark 5).

Example 32. Consider the holomorphic function $f_{1}$ defined by

$$
f_{1}(z)=-(1-z)^{2} \cdot \frac{2+\sqrt{\frac{1+z}{1-z}}}{1+\sqrt{\frac{1+z}{1-z}}} \cdot \frac{1+z}{1-z} .
$$

One can check by using the Berkson-Porta representation formula that $f_{1} \in \mathscr{G}[1]$. Moreover, $f_{1}^{\prime}(1)=2$, i.e., the semigroup $S_{1}$ generated by $f_{1}$ is of hyperbolic type. In addition, $f_{1}(-1)=0$ and $f_{1}^{\prime}(-1)=-4$. So, by Theorem 1 in [24], $S_{1}$ has a BFID. Using Theorem 3 in [24], one can find a univalent function $\varphi$ which maps $\Delta$ onto the maximal BFID $\Omega$ conformally. Namely,

$$
\varphi(z)=\frac{(w-1)^{2}-1}{(w-1)^{2}+1}, \quad \text { where } \quad w=\sqrt{1+\sqrt{\frac{1+z}{1-z}}} .
$$

In Figure (a) we present the vector field of the flow $S_{1}$, as well as the location of the h-type maximal BFID for $S_{1}$ in the unit disk $\Delta$.

Example 33. Consider now another semigroup generator:

$$
f_{2}(z)=-(1-z)^{2} \frac{1-z^{2}}{1+z^{2}} \text {. }
$$




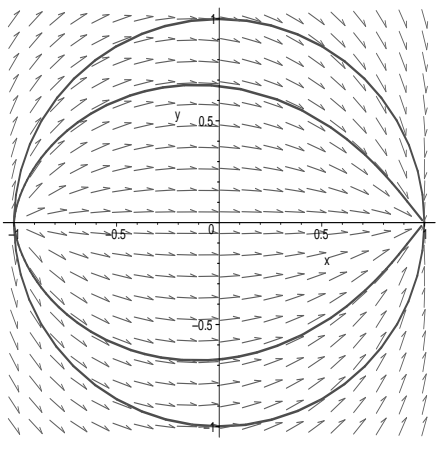

(a) h-type maximal BFID

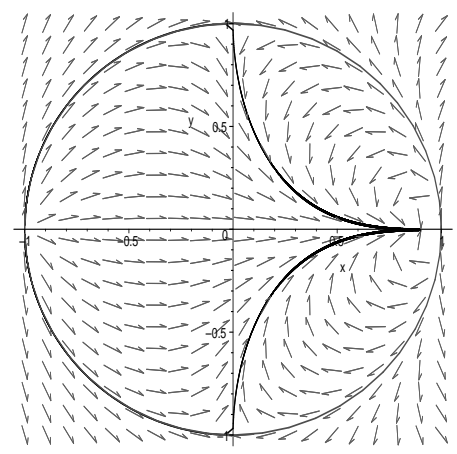

(b) BFIDs of h-type and p-type

Since $f_{2}^{\prime}(1)=0$, we conclude that the semigroup $S_{2}=\left\{F_{t}\right\}_{t>0}$ generated by $f_{2}$ is of parabolic type. Using Theorems 1 and 3 in [2], and Theorem 34 and Remark 35 below, one can show that $S_{2}$ has BFIDs of both types: two domains of p-type and a domain of h-type. More precisely, in this case the closure of the union of these three BFIDs is the whole disk $\Delta$. In other words, there are only two trajectories $\left\{F_{t}\left(z_{i}\right)\right\}_{t>0}, i=1,2$, that cannot be extended to all the values of $t \in \mathbf{R}$. In Figure (b) we show the maximal BFIDs of both types.

The following theorem is mostly based on the results established in [11] and [24] by using different techniques. For the sake of completeness we provide a proof which combines them.

Theorem 34. Let $f \in \mathscr{G}[1]$ and let $S=\left\{F_{t}\right\}_{t \geq 0}$ be the semigroup generated by $f$. The following assertions are equivalent:

(i) there is a point $z \in \Delta$ such that $F_{t}(z) \in \Delta$ for all $t \in \mathbf{R}$ and the point $\zeta=\lim _{t \rightarrow-\infty} F_{t}(z)$ is a boundary regular null point of $f$, i.e., $f^{\prime}(\zeta)=\angle \lim _{z \rightarrow \zeta} f(z)$ is finite;

(ii) the image $h(\Delta)$, where $h \in \Sigma[1]$ is defined by

$$
h(z)=-\int_{0}^{z} \frac{d z}{f(z)},
$$

contains a horizontal strip (or a horizontal half-plane);

(iii) there is a simply connected open subset $\Omega \subset \Delta$ such that $\left.F_{t}\right|_{\Omega}$ is a group of automorphisms of $\Omega$;

(iv) there are real numbers $a$ and $b$ such that the differential equation

$$
\left[a\left(z^{2}-1\right)+i b(1-z)^{2}\right] \varphi^{\prime}(z)=f(\varphi(z))
$$

has a nonconstant solution $\varphi \in \operatorname{Hol}(\Delta)$.

Moreover, in this case $\varphi$ is univalent and $\Omega=\varphi(\Delta)$ is a BFID for $S$.

Proof. The fundamental part of the theorem is the equivalence of assertions (i) and (ii) which has been shown in [11] (both the parabolic and hyperbolic cases) and [24] (the hyperbolic case). 
Assertion (iii) is a direct consequence of (ii). Indeed, if $k$ is a Riemann mapping of $\Delta$ onto a horizontal strip (or a half-plane) contained in $h(\Delta)$, then the function $\varphi=h^{-1} \circ k$ is a univalent self-mapping of $\Delta$ and $\Omega=\varphi(\Delta)$ is a BFID for $S$. Indeed, the family $\left\{G_{t}\right\}_{t \in \mathbf{R}}$ defined by $G_{t}(z)=k^{-1}(k(z)+t), t \in \mathbf{R}$, forms a one-parameter group of hyperbolic (or parabolic) automorphisms of $\Delta$. On the other hand,

$$
\varphi\left(G_{t}(z)\right)=h^{-1}(k(z)+t)=h^{-1}(h(\varphi(z))+t)=F_{t}(\varphi(z)) .
$$

That is, $\varphi$ is, in fact, an inner conjugating function for $\left\{F_{t}\right\}_{t \geq 0}$ and $\left\{G_{t}\right\}_{t \in \mathbf{R}}$.

To prove the implication (iii) $\Rightarrow$ (iv), we first observe that up to an automorphism we can assume that $\tau=1$ is the Denjoy-Wolff point of the group $\left\{G_{t}\right\}_{t \in \mathbf{R}}$ defined by (42). Then it follows from (44) and Lindelöf's Theorem (see, for example, [10] and [38]) that $\angle \lim _{z \rightarrow 1} \varphi(z)=1$. In addition, it is well known that the generator $g: \Delta \mapsto \mathbf{C}$ of the group $\left\{G_{t}\right\}_{t \in \mathbf{R}}$ must be of the form

$$
g(z)=a\left(z^{2}-1\right)+i b(z-1)^{2}
$$

with $a \geq 0$ and $b \in \mathbf{R}$. Moreover, $a=0$ if and only if $\left\{G_{t}\right\}_{t \geq 0}$ is of parabolic type. In the last case, for each $z \in \Delta$ we have $\lim _{t \rightarrow \pm \infty} G_{t}(z)=1$.

If $a \neq 0(a>0)$, then the point $\eta=\frac{i b-a}{i b+a}$ is a repelling point of the group $\left\{G_{t}\right\}_{t \in \mathbf{R}}$, i.e., $\lim _{t \rightarrow-\infty} G_{t}(z)=\eta$ for all $z \in \Delta$ (or, which is one and the same, $g(\eta)=0$ and $\left.g^{\prime}(\eta)=-g^{\prime}(1)<0\right)$. In this case, up to an automorphism of $\Delta$ we can assume that $\eta=-1$ or, which is one and the same, $b=0$. In any case, differentiating (43) at $t=0$, we obtain that $\varphi$ must satisfy the differential equation

$$
\varphi^{\prime}(z) \cdot g(z)=f(\varphi(z))
$$

where $g$ is given by (47).

Finally, we show that assertion (iv) implies (ii) as well as (iii). Let $\varphi$ be a holomorphic solution of the differential equation (48) with $|\varphi(z)|<1$, where $g \in$ $\operatorname{Hol}(\Delta, \mathbf{C})$ is given by $(47)$. We claim that $\varphi$ is univalent and is a conjugating function for the semigroup $S=\left\{F_{t}\right\}_{t \geq 0}$ with a group $\left\{G_{t}\right\}_{t \in \mathbf{R}}$ of automorphisms of $\Delta$.

To this end, let us consider the function $h \in \operatorname{Hol}(\Delta, \mathbf{C})$ defined by (45). Recall that since $f(z) \neq 0, z \in \Delta$, the function $h$ is holomorphic in $\Delta$ and satisfies Abel's functional equation (4). If we define the function $k \in \operatorname{Hol}(\Delta, \mathbf{C})$ by $k(z)=h(\varphi(z)), z \in \Delta$, then we have by $(45)$

$$
k^{\prime}(z)=h^{\prime}(\varphi(z)) \cdot \varphi^{\prime}(z)=-\frac{\varphi^{\prime}(z)}{f(\varphi(z))} .
$$

On the other hand, it follows from (48) that

$$
k^{\prime}(z)=-\frac{1}{g(z)},
$$

Thus we get from (47)

$$
k(z)= \begin{cases}\frac{1}{2 a} \log \frac{\eta-z}{1-z}+C, & a \neq 0, \\ \frac{1}{b} \frac{i}{1-z}+C, & a=0,\end{cases}
$$


where $C=h(\varphi(0))$ and $\eta=\frac{i b-a}{i b+a}$. First, this formula shows that

$$
\varphi=h^{-1} \circ k
$$

must be univalent on $\Delta$. Second, $h(\Delta)$ must contain at least a nonempty horizontal strip or even a horizontal half-plane $k(\Delta)=h(\varphi(\Delta))$. In fact, $h(\Delta)$ cannot contain a half-plane if $S=\left\{F_{t}\right\}_{t \geq 0}$ is of hyperbolic type (see [11]).

In any case, the trajectory $\widetilde{F}_{t}(w)=h^{-1}(h(w)+t)$ is well defined for each $w \in \Omega$ and all $t \in \mathbf{R}$. Hence, the family $\left\{\widetilde{F}_{t}\right\}_{t \in \mathbf{R}}$ forms a group on $\Omega=\varphi(\Delta)=h^{-1}(k(\Delta))$ which is an extension of the semigroup $S=\left\{F_{t}\right\}_{t \geq 0}$ on $\Omega$. Then setting again

$$
G_{t}(z)=\varphi^{-1}\left(F_{t}(\varphi(z))\right.
$$

we obtain a conjugation group $\left\{G_{t}\right\}_{t \geq 0}$ of automorphisms on $\Delta$, the generator of which is exactly $g(z)=a\left(z^{2}-1\right)+i \bar{b}(z-1)^{2}$. So, $\Omega=\varphi(\Delta)$ is of $h$-type if and only if $a \neq 0$ and is of $p$-type otherwise $(a=0, b \neq 0)$. Similarly as in [11] and [24] (or by using Lemma 2 in [37], p. 162), one shows that for each $w \in \Omega$, the limit $\varphi(\eta)=\lim _{t \rightarrow-\infty} F_{t}(w)$ exists and belongs to $\partial \Delta$. In particular, if $\eta=1$, then $\varphi(1)$ must also be 1 .

Remark 35. Actually, one can distinguish between the following two situations.

(A) The following assertions are equivalent:

(a) there is a boundary regular point $\zeta \in \partial \Delta$ of the generator $f$ which is different from the Denjoy-Wolff point $\tau=1$;

(b) $h(\Delta)$ contains a horizontal strip;

(c) there are numbers $a \neq 0$ and $b \in \mathbf{R}$ such that equation (46) has a solution $\varphi$ for which $\varphi(\Delta)=\Omega$ is a BFID of $h$-type, i.e., $S=\left\{F_{t}\right\}$ is a conjugate of a group of hyperbolic automorphisms and $\partial \Omega \cap \partial \Delta \ni \zeta \neq 1$.

(B) The following assertions are equivalent:

(a') there is a point $z \in \Delta$ such that $\left\{F_{t}(z)\right\}$ is well defined for all $t \in \mathbf{R}$ and

$$
\lim _{t \rightarrow-\infty} F_{t}(z)=1
$$

(b') $h(\Delta)$ contains a half-plane;

(c') equation (46) with $a=0$ and $b \neq 0$ has a nonconstant solution $\varphi$ such that $\varphi(\Delta)=\Omega$ is a BFID of $p$-type, i.e., $S=\left\{F_{t}\right\}$ is a conjugate of a group of parabolic automorphisms and $\partial \Omega \cap \partial \Delta \ni 1$.

3.2. The classes $\Sigma^{\alpha}\left(\mathscr{G}^{\alpha}\right)$ and corners of domains. Now we will concentrate on geometric properties of a backward flow invariant domain $\Omega$ of $p$-type. We show, in particular, that under some smoothness conditions $\Omega$ has a corner of opening $\pi / \alpha$ (at the point $z=1$ ) if and only if $f \in \mathscr{G}^{\alpha}[1]$ (respectively, $h \in \Sigma^{\alpha}[1]$ ) for some $\alpha \in(1,2]$.

We have proved that if $\Omega$ is of $p$-type, then for all $w \in \Omega$,

$$
\lim _{t \rightarrow-\infty} F_{t}(w)=1 \text {. }
$$

As we have already mentioned, it follows from Theorem 2.4 in [11] that if for at least one $w \in \Delta$ the trajectory $\left\{F_{t}(w)\right\}_{t \in \mathbf{R}}$ is well defined and condition (52) holds, then there is a horizontal half-plane contained in $h(\Delta)$. Thus condition (52) is a necessary and sufficient condition for existence of a BFID $\Omega$ of $p$-type. 
So, we assume now that for some $z \in \Delta$, the straight line $h(z)+t$ is contained in $h(\Delta)$ for all $t \in \mathbf{R}$ and

$$
\lim _{t \rightarrow \pm \infty} h^{-1}(h(z)+t)=1 .
$$

If, in addition, $h \in \Sigma^{\alpha}[1]$, i.e.,

$$
\lim _{z \rightarrow 1}(1-z)^{1+\alpha} h^{\prime}(z)=\mu \neq 0, \infty
$$

then we have

$$
\lim _{z \rightarrow 1} \frac{f(z)}{(1-z)^{1+\alpha}}=a=-\frac{1}{\mu} \neq 0, \infty
$$

where, $\alpha \in(0,2]$ and $f(z)=-\frac{1}{h^{\prime}(z)}$ is the generator of the semigroup $u(t, z):=$ $h^{-1}(h(z)+t)$. It follows now from the Cauchy problem (see also Theorem 12 and its proof)

$$
\left\{\begin{array}{l}
\frac{d u}{d t}+f(u)=0 \\
u(t, z)=z \in \Delta
\end{array}\right.
$$

that

or

$$
\frac{d u}{(1-u)^{1+\alpha}}=-\frac{f(u) d t}{(1-u)^{1+\alpha}}
$$

$$
\frac{1}{\alpha} \cdot \frac{1}{t} \frac{1}{(1-u)^{\alpha}}=-\frac{1}{t} \int_{0}^{t} \frac{f(u) d t}{(1-u)^{1+\alpha}}+\frac{1}{t(1-z)^{\alpha}} .
$$

Hence,

or

$$
\lim _{t \rightarrow \pm \infty} \frac{1}{t(1-u(t, z))^{\alpha}}=\frac{1}{\mu} \cdot \alpha
$$

$$
\lim _{t \rightarrow \pm \infty} t(1-u(t, z))^{\alpha}=\frac{\mu}{\alpha} \neq 0, \infty
$$

Therefore,

$$
\lim _{t \rightarrow \infty} \arg (1-u(t, z))=\frac{1}{\alpha} \arg \mu
$$

while

$$
\lim _{t \rightarrow-\infty} \arg (1-u(t, z))=\frac{1}{\alpha}(\arg \mu \pm \pi)
$$

Consequently,

$$
\left|\lim _{t \rightarrow \infty} \arg (1-u(t, z))-\lim _{t \rightarrow-\infty} \arg (1-u(t, z))\right|=\frac{\pi}{\alpha} .
$$

Since the trajectory $\{u(t, z)\}_{t \in \mathbf{R}}$ belongs to $\Delta$, we see that $\alpha$ must be greater than or equal to 1.

This leads us to the following assertion. 
Proposition 36. Let $f \in \mathscr{G}^{\alpha}[1]$ with

$$
\lim _{z \rightarrow 1} \frac{f(z)}{(1-z)^{1+\alpha}}=a \neq 0, \infty
$$

and let $S=\left\{F_{t}\right\}_{t \geq 0}$ be the semigroup generated by $f$. Assume that for a point $z \in \Delta$, the trajectory $\left\{F_{t}(z)\right\}_{t \geq 0}$ can be extended to the whole real axis and

$$
\lim _{t \rightarrow-\infty}\left(1-F_{t}(z)\right)=1 \text {. }
$$

Then $\alpha \in[1,2]$ and

(i) $|\arg (-a)|=\frac{\pi}{2}(2-\alpha)$;

(ii) the trajectory $\left\{F_{t}(z)\right\}_{t<0}$ converges tangentially to the point $z=1$ as $t$ tends to $-\infty$. Moreover, if $\arg (-a) \neq 0$, then

$$
\lim _{t \rightarrow-\infty} \arg \left(1-F_{t}(z)\right)=(\operatorname{sign} \arg (-a)) \frac{\pi}{2} ;
$$

(iii) there is a BFID $\Omega \subset \Delta$ of $p$-type which has a corner of opening $\pi \gamma$ at the point $z=1$ with $\gamma=\frac{1}{\alpha}$.

Proof. Since $\left\{F_{t}(z)\right\}_{t \in \mathbf{R}} \subset \Delta$, we have

$$
-\frac{\pi}{2} \leq \lim _{t \rightarrow-\infty} \arg \left(1-F_{t}(z)\right) \leq \frac{\pi}{2} .
$$

Assume that $\arg (-a)(=-\arg \mu)<0$. Since $\alpha \leq 2$, we have

$$
\frac{1}{\alpha}(\arg \mu+\pi)>\frac{\pi}{\alpha} \geq \frac{\pi}{2} .
$$

Hence the sign $(+)$ in (59) is impossible, and we have by (59) and (61),

$$
\lim _{t \rightarrow-\infty} \arg \left(1-F_{t}(z)\right)=\frac{1}{\alpha}(\arg \mu-\pi) \geq-\frac{\pi}{2} .
$$

This, in turn, implies that

$$
\arg \mu \geq \pi-\frac{\pi}{2} \alpha=\frac{\pi}{2}(2-\alpha) .
$$

But it follows from Theorem 12 and (55) that

$$
\arg \mu(=|\arg (-a)|) \leq \frac{\pi}{2}(2-\alpha) .
$$

Thus

$$
\arg \mu=\frac{\pi}{2}(2-\alpha) .
$$

So we again obtain from (59) that

$$
\lim _{t \rightarrow-\infty} \arg \left(1-F_{t}(z)\right)=\frac{1}{\alpha}\left[\pi-\frac{\pi \alpha}{2}-\pi\right]=-\frac{\pi}{2} .
$$

In a similar way, we prove our assertion if $\arg (-a)(=-\arg \mu)>0$. If $\arg \mu=0$, then

$$
\lim _{t \rightarrow-\infty} \mid \arg \left(1-F_{t}(z) \mid=\frac{\pi}{\alpha} \geq \frac{\pi}{2}\right.
$$

and we get $\alpha=2$.

Thus we have also proved the following assertions. 
Corollary 37. If the assumptions of Proposition 36 hold, then

$$
\lim _{t \rightarrow \infty} \arg \left(1-F_{t}(z)\right)=-\operatorname{sgn} \arg (-a) \cdot \frac{\pi(2-\alpha)}{2 \alpha} .
$$

Thus, the trajectory $\left\{F_{t}(z)\right\}_{t \geq 0}$ is tangential to the unit circle at the point $z=1$ if and only if $\alpha=1$. This trajectory is tangential to the real axis at the point $z=1$ if and only if $\alpha=2$.

Corollary 38. Let $h \in \Sigma^{\alpha}[1]$ with $\mu=\lim (1-z)^{1+\alpha} h^{\prime}(z)$. The following assertions are equivalent:

(i) there is $z \in \Delta$ such that the point $h(z)+t \in h(\Delta)$ for all $t \in \mathbf{R}$ and

$$
\lim _{t \rightarrow-\infty} h^{-1}(h(z)+t)=1
$$

(ii) $h(\Delta) \supset k(\Delta)$ (a half-plane), where

$$
k(z)=\frac{i b z}{1-z}+c
$$

for some $b, c \in \mathbf{R}$.

Moreover, in this case:

(a) $\alpha \in[1,2]$;

(b) if $\alpha \in[1,2)$, then there is no horizontal half-plane $H \subset h(\Delta)$ such that $H \cap k(\Delta)=\emptyset$ and $b \cdot \arg \mu>0$.

Proof. In fact, we only have to prove assertion (b). Indeed, if $\alpha \neq 2$, then there is only one maximal BFID $\Omega$ of $p$-type, since its angle of opening at $z=1$ is $\frac{\pi}{\alpha}>\frac{\pi}{2}$. Let now $\varphi=h^{-1} \circ k$ be a conformal mapping on $\Omega$. We claim that the limit

$$
\angle \lim _{z \rightarrow 1} \frac{1-z}{[1-\varphi(z)]^{\alpha}}
$$

exists finitely and is different from zero.

To prove this, we note that the group $\left\{G_{t}\right\}_{t \in \mathbf{R}}$ is generated by $g, g(z)=i b(z-$ $1)^{2}$, which belongs to the class $\mathscr{G}^{1}[1]$ with $\lim _{z \rightarrow 1} \frac{g(z)}{(1-z)^{2}}=i b$. In addition, since $F_{t}(\varphi(z))=\varphi\left(G_{t}(z)\right), t \geq 0, z \in \Delta$, we have by formula $(57)$,

$$
\lim _{t \rightarrow \infty} \frac{1-G_{t}(z)}{\left[1-\varphi\left(G_{t}(z)\right)\right]^{\alpha}}=\lim _{t \rightarrow \infty} \frac{t\left(1-G_{t}(z)\right)}{t\left[1-F_{t}(\varphi(z))\right]^{\alpha}}=-\frac{\alpha}{\mu i b} .
$$

Since the function

$$
\frac{(1-z)^{1 / \alpha}}{1-\varphi(z)}
$$

does not admit negative real values, we get by the generalized Lindelöf Theorem (see Theorem 2.20 in [10], p. 42) that

$$
\angle \lim _{z \rightarrow 1} \frac{1-z}{[1-\varphi(z)]^{\alpha}}=-\frac{\alpha}{\mu i b} \neq 0, \infty .
$$

Hence the limit

$$
m:=\angle \lim _{z \rightarrow 1} \frac{(1-z)^{1 / \alpha}}{1-\varphi(z)}
$$


is also finite and different from zero. Since the function $\frac{1}{1-\varphi(z)}$ is of positive real part, it follows from Lemma 13 that

$$
|\arg m| \leq \frac{\pi}{2}\left(1-\frac{1}{\alpha}\right)
$$

or

$$
\left|\arg m^{\alpha}\right|=\left|\arg \left(-\frac{\alpha}{\mu i b}\right)\right|=\left|\frac{\pi}{2}-\arg (\mu b)\right| \leq \frac{\pi}{2}(\alpha-1) .
$$

This implies that if $b>0$, then also $\arg \mu>0$ and conversely: if $b<0$, then $\arg \mu>0$, and we are done.

In some sense a converse assertion also holds.

Theorem 39. Let $h \in \Sigma[1]$ and suppose that $F_{t}(z)=h^{-1}(h(z)+t)$ can be extended for some $z \in \Delta$ to the whole real axis with

$$
\lim _{t \rightarrow-\infty} F_{t}(z)=1 \text {. }
$$

In other words, there is a nonempty BFID $\Omega$ of p-type. Suppose further that $\partial \Omega$ has a Dini-smooth corner of opening $\pi \gamma$ at the point $z=1$. The following assertions hold:

(i) $h \in \Sigma_{A}^{\alpha}[1]$, with $\alpha=\frac{1}{\gamma}$ and $\mu:=\angle \lim _{z \rightarrow 1}(1-z)^{1+\alpha} h^{\prime}(z) \neq 0, \infty$;

(ii) $\frac{1}{2} \leq \gamma \leq 1$, hence the angle of the opening cannot be less than $\frac{\pi}{2}$ and $|\arg \mu| \leq$ $\frac{\pi(2 \gamma-1)}{2 \gamma}$

(iii) the angular limit of the inverse Visser-Ostrovskii quotient

$$
\angle \lim _{z \rightarrow 1} \frac{h(z)}{(z-1) h^{\prime}(z)}
$$

exists and equals $\gamma$.

Proof. We have already shown that there is a Riemann conformal mapping from $\Delta$ onto $\Omega$ such that $\varphi(1)=1$ and $\varphi$ satisfies the differential equation

$$
i b(1-z)^{2} \varphi^{\prime}(z)=f(\varphi(z)),
$$

where $f=-\frac{1}{h} \in \mathscr{G}[1]$. It follows from Theorem 3.9 in [30], p. 52, that the limits

$$
\lim _{z \rightarrow 1} \frac{1-\varphi(z)}{(1-z)^{\gamma}}=m \neq 0, \infty
$$

and

$$
\lim _{z \rightarrow 1} \frac{\varphi^{\prime}(z)}{(1-z)^{\gamma-1}}=n \neq 0, \infty
$$

exist.

In addition, as in the proof of this theorem, one can show that the limit of the Visser-Ostrovskii quotient

$$
\frac{(1-z) \varphi^{\prime}(z)}{1-\varphi(z)}
$$


exists and equals $\gamma$ :

$$
\lim _{z \rightarrow 1} \frac{(1-z) \varphi^{\prime}(z)}{1-\varphi(z)}=\frac{n}{m}=\gamma .
$$

Taking some $\alpha>0$, consider the limit

$$
a:=\lim _{z \rightarrow 1} \frac{f(\varphi(z))}{(1-\varphi(z))^{1+\alpha}} .
$$

We have by (63),

$$
a=i b \lim _{z \rightarrow 1} \frac{(1-z)^{2} \varphi^{\prime}(z)}{(1-\varphi(z))^{1+\alpha}}=i b \lim _{z \rightarrow 1} \frac{(1-z) \varphi^{\prime}(z)}{1-\varphi(z)} \cdot \lim _{z \rightarrow 1} \frac{1-z}{(1-\varphi(z))^{\alpha}} .
$$

Hence we infer from (64) and (66) that the limit in (67) exists and is different from zero if and only if $\alpha=\frac{1}{\gamma}$. If this is indeed the case, we have

$$
-\frac{1}{\mu}=a=\frac{i b}{\alpha m^{\alpha}}=\frac{i b \gamma}{m^{\alpha}} .
$$

Furthermore, if we represent $f$ by the Berkson-Porta representation formula $f(z)=-(1-z)^{2} p(z)$, then we see that the limit

$$
\lim _{\substack{w \rightarrow 1 \\ w=\varphi(z) \in \Omega}} \frac{p(w)}{(1-w)^{\alpha-1}}=-a
$$

exists finitely and is different from zero. Since $\gamma \leq 1$, we have $\alpha \geq 1$. In addition, it follows from a rigidity theorem in [19] that $\alpha \leq 2$. So, $0 \leq \alpha-1 \leq 1$. For $\alpha \in[1,2)$ we have

$$
\left|\arg \frac{p(z)}{(1-z)^{\alpha-1}}\right|=|\arg p(z)-(\alpha-1) \arg (1-z)| \leq \frac{\pi}{2} \alpha<\pi
$$

Thus the function $\frac{p(z)}{(1-z)^{1-\alpha}}$ does not admit negative real values. Then it follows from (69) and the generalized Lindelöf Theorem (see Theorem 2.20 in [10], p. 42) that

$$
\lim _{z \rightarrow 1} \frac{p(z)}{(1-z)^{\alpha-1}}=-a
$$

whenever $1 \leq \alpha<2$.

If $\alpha=2$, then one can show by using the Riesz-Herglotz representation of $p$ that the function $\frac{p(z)}{1-z}$ is bounded on each nontangential approach region in $\Delta$ with vertex at 1 . So, one can again apply Lindelöf's Theorem to show that the angular limit

$$
\angle \lim _{z \rightarrow 1} \frac{p(z)}{1-z}=-a
$$

exists. Moreover, in this case $a$ is a negative real number. Setting $\mu=-\frac{1}{a}$, we see that the limit

$$
\angle \lim _{z \rightarrow 1}(1-z)^{1+\alpha} h^{\prime}(z)=\left(=-\angle \lim _{z \rightarrow 1} \frac{(1-z)^{\alpha-1}}{p(z)}\right)=\mu \neq 0, \infty .
$$


So $h \in \Sigma^{\alpha}[1]$, and by Theorem 10,

$$
|\arg \mu| \leq \frac{\pi}{2}(2-\alpha)=\frac{\pi(2 \gamma-1)}{2 \gamma}
$$

Assertions (i) and (ii) are proved.

To prove assertion (iii), we again consider equation (63), rewriting it in the form

$$
h^{\prime}(\varphi(z)) \cdot \varphi^{\prime}(z)=\frac{-1}{i b(1-z)^{2}} .
$$

Integrating this equation, we get

$$
h(\varphi(z))=k(z):=\frac{-1}{i b} \frac{z}{1-z}+h(\varphi(0)) .
$$

Since $\varphi$ is an inner conjugating function for the semigroup $S=\left\{F_{t}\right\}_{t \geq 0}$ and the group $\left\{G_{t}\right\}_{t \in R}$, we have, for all $w \in \Omega$,

$$
F_{t}(w)=\varphi\left(G_{t}\left(\varphi^{-1}(w)\right)\right)
$$

or

Consequently,

$$
\varphi^{-1}\left(F_{t}(w)\right)=G_{t}\left(\varphi^{-1}(w)\right)
$$

$$
\lim _{t \rightarrow \infty} \varphi^{-1}\left(F_{t}(w)\right)=1 .
$$

In other words, there is a trajectory converging to 1 such that the limit of $\varphi^{-1}$ along this trajectory is also 1 . Therefore, we have from (64) that

$$
\lim _{t \rightarrow \infty} \frac{\left(1-F_{t}(w)\right)^{\alpha}}{1-\varphi^{-1}\left(F_{t}(w)\right)}=\lim _{t \rightarrow \infty} \frac{\left(1-\varphi\left(G_{t}\left(\varphi^{-1}(w)\right)\right)^{\alpha}\right.}{1-G_{t}\left(\varphi^{-1}(w)\right)}=m^{\alpha} .
$$

On the other hand, by direct calculations from (71) we get

$$
1-\varphi^{-1}\left(F_{t}(w)\right)=1-k^{-1}\left(h\left(F_{t}(w)\right)\right)=\frac{1}{1-i b\left(h\left(F_{t}(w)\right)-h(\varphi(0))\right.} .
$$

Therefore, using (72), we get

$$
\begin{aligned}
m^{\alpha} & =\lim _{t \rightarrow \infty}\left(1-F_{t}(w)\right)^{\alpha}\left[1-i b\left(h\left(F_{t}(w)\right)-h(\varphi(0))\right)\right]= \\
& =-i b \lim _{t \rightarrow \infty}\left(1-F_{t}(w)\right)^{\alpha} h\left(F_{t}(w)\right) .
\end{aligned}
$$

Now we observe that since $h$ is univalent and $\alpha \geq 1$, the function $(1-z)^{\alpha} h(z)$ is bounded on each nontangential approach region $\{z \in \mathbf{C}:|1-z| \leq r(1-|z|), r>1\}$. Indeed, it follows from Koebe's inequality (see, for example, [18] and [30]) that

$$
|1-z|^{\alpha}|h(z)| \leq\left|h^{\prime}(0)\right|(1+|z|) \cdot \frac{|1-z|}{1-|z|} \cdot|1-z|^{\alpha-1} \leq 2 r\left|h^{\prime}(0)\right||1-z|^{\alpha-1} .
$$

Therefore, again by the Lindelöf's theorem and (73), we arrive at

$$
\angle \lim _{z \rightarrow 1}(1-z)^{\alpha} h(z)=-\frac{m^{\alpha}}{i b} .
$$

Now it follows from (68) that

$$
\angle \lim _{z \rightarrow 1} \frac{h(z)}{(z-1) h^{\prime}(z)}=-\angle \lim _{z \rightarrow 1} \frac{(1-z)^{\alpha} h(z)}{(1-z)^{1+\alpha} h^{\prime}(z)}=\gamma .
$$

Theorem 44 is proved. 
Acknowledgments. Dmitry Khavinson's research was supported by a grant from the National Science Foundation. Simeon Reich was partially supported by the Fund for the Promotion of Research at the Technion and by the Technion President's Research Fund. This research is part of the European Science Foundation Networking Programme HCAA. All of the authors are very grateful to the referee for a very detailed and helpful report.

\section{References}

[1] Abate, M.: The infinitesimal generators of semigroups of holomorphic maps. - Ann. Mat. Pura Appl. 161, 1992, 167-180.

[2] Aharonov, D., M. Elin, S. Reich, and D. Shoikhet: Parametric representations of semicomplete vector fields on the unit balls in $\mathbf{C}^{n}$ and in Hilbert space. - Atti Accad. Naz. Lincei 10, 1999, 229-253.

[3] Baracco, L., D. Zaitsev, and G. Zampieri: A Burns-Krantz type theorem for domains with corners. - Math. Ann. 336, 2006, 491-504.

[4] Berkson, E., and H. Porta: Semigroups of analytic functions and composition operators. Michigan Math. J. 25, 1978, 101-115.

[5] Bourdon, P.S., and J. H. Shapiro: Cyclic phenomena for composition operators. - Mem. Amer. Math. Soc. 125:596, 1997.

[6] Bracci, F., R. Tauraso, and F. VlacCi: Identity principles for commuting holomorphic self-maps of the unit disc. - J. Math. Anal. Appl. 270, 2002, 451-473.

[7] Burns, D. M., and S. G. Krantz: Rigidity of holomorphic mappings and a new Schwarz lemma at the boundary. - J. Amer. Math. Soc. 7, 1994, 661-676.

[8] Ciozda, K.: Sur la classe des fonctions convexes vers l'axe réel négatif. - Bull. Acad. Polon. Sci. Sér. Sci. Math. 27, 1979, 255-261.

[9] CiozDa, K.: Sur quelques problémes extrèmaux dans les classes des fonctions convexes vers l'axe réel négatif. - Ann. Polon. Math. 38, 1980, 311-317.

[10] Collingwood, E. F., and A. J. Lohwater: The theory of cluster sets. - Cambridge Univ. Press, Cambridge, 1966.

[11] Contreras, M. D., and S. Díaz-Madrigal: Analytic flows on the unit disk: angular derivatives and boundary fixed points. - Pacific J. Math. 222, 2005, 253-286.

[12] Contreras, M.D., S. Díaz-Madrigal, and Ch. Pommerenke: On boundary critical points for semigroups of analytic functions. - Math. Scand. 98, 2006, 125-142.

[13] Contreras, M. D., S. Díaz-Madrigal, and Ch. Pommerenke: Some remarks on the Abel equation in the unit disk. - J. London Math. Soc. (2) 75, 2007, 623-634.

[14] Contreras, M. D., S. Díaz-Madrigal, and Ch. Pommerenke: Second angular derivatives and parabolic iteration in the unit disk. - Trans. Amer. Math. Soc. 362, 2010, 357-388.

[15] Cowen, C. C.: Iteration and the solution of functional equations for functions analytic in the unit disk. - Trans. Amer. Math. Soc. 265, 1981, 69-95.

[16] Cowen, C. C., and B. D. MacCluer: Composition operators on spaces of analytic functions. - CRC Press, Boca Raton, FL, 1995.

[17] Díaz-Madrigal, S.: Personal communications.

[18] Duren, P.: Univalent functions. - Springer, New York, 1983.

[19] Elin, M., M. Levenshtein, S. Reich, and D. Shoikhet: Commuting semigroups of holomorphic mappings. - Math. Scand. 103, 2008, 295-319.

[20] Elin, M., and D. Shoikhet: Dynamic extension of the Julia-Wolff-Carathéodory theorem. Dynam. Systems Appl. 10, 2001, 421-437. 
[21] Elin, M., and D. Shoikhet: Univalent functions of proper contractions spirallike with respect to a boundary point. - In: Multidimensional Complex Analysis, Krasnoyarsk, 2002, 28-36.

[22] Elin, M., and D. Shoikheт: Angle distortion theorems for starlike and spirallike functions with respect to a boundary point. - Int. J. Math. Math. Sci. 81615, 2006, 1-13.

[23] Elin, M., D. Shoikhet, and F. Yacobzon: Linearization models for parabolic type semigroups. - J. Nonlinear Convex Anal. 9, 2008, 205-214.

[24] Elin, M., D. Shoikhet, and L. Zalcman: A flower structure of backward flow invariant domains for semigroups. - C. R. Math. Acad. Sci. Paris 346, 2008, 293-296.

[25] Goodman, A. W.: Univalent functions, Vol. I and II. - Mariner Publ. Co., Tampa, FL, 1983.

[26] Harris, T.E.: The theory of branching processes. - Springer-Verlag, Berlin-GöttingenHeidelberg, 1963.

[27] Hengartner, W., and G. Schober: On schlicht mappings to domains convex in one direction. - Comm. Math. Helv. 45, 1970, 303-314.

[28] Kriete, T. L., and B. D. MaCCluer: A rigidity theorem for composition operators on certain Bergman spaces. - Michigan Math. J. 42, 1995, 379-386.

[29] Lecko, A.: On the class of functions convex in the negative direction of the imaginary axis. J. Aust. Math. Soc. 73, 2002, 1-10.

[30] Pommerenke, Ch.: Boundary behavior of conformal maps, Springer, New York, 1992.

[31] Reich, S., and D. Shoikhet: Generation theory for semigroups of holomorphic mappings in Banach spaces. - Abstr. Appl. Anal. 1, 1996, 1-44.

[32] Reich, S., and D. Shoikhet: Semigroups and generators on convex domains with the hyperbolic metric. - Atti Accad. Naz. Lincei 8, 1997, 231-250.

[33] Reich, S., and D. Shoikhet: The Denjoy-Wolff theorem. - Ann. Univ. Mariae Curie-Skłodowska Sect. A 51, 1997, 219-240.

[34] Reich, S., and D. Shoikhet: Metric domains, holomorphic mappings and nonlinear semigroups. - Abstr. Appl. Anal. 3, 1998, 203-228.

[35] Reich, S, and D. Shoikhet: Nonlinear semigroups, fixed points, and geometry of domains in Banach spaces. - Imperial College Press, London, 2005.

[36] Robertson, M. S.: Analytic functions starlike in one direction. - Amer. J. Math. 58, 1936, $465-472$.

[37] Shapiro, J. H.: Composition operators and classical function theory. - Springer-Verlag, New York, 1993.

[38] Shoikhet, D.: Semigroups in geometrical function theory. - Kluwer, Dordrecht, 2001.

[39] Shoikнeт, D.: Representations of holomorphic generators and distortion theorems for spirallike functions with respect to a boundary point. - Int. J. Pure Appl. Math. 5, 2003, 335-361.

[40] Shoikhet, D.: Koenigs type linearization models and asymptotic behavior of one-parameter semigroups. - Contemp. Math. 21, 2007, 149-166.

[41] Shoikhet, D.: Another look at the Burns-Krantz Theorem, - J. Anal. Math. 19, 2008, 19-42.

[42] Siskakis, A.: Semigroups of composition operators on spaces of analytic functions, a review. - In: Studies on Composition Operators (Laramie, WY, 1996), Contemp. Math. 213, 1998, $229-252$.

[43] Tauraso, R.: Commuting holomorphic maps of the unit disc. - Ergodic Theory Dynam. Systems 24, 2004, 945-953.

[44] Valiron, G: Fonctions analytiques. - Presses Univ. France, Paris, 1954. 